\title{
Charged pion condensation and duality in dense and hot chirally and isospin asymmetric quark matter in the framework of the $\mathrm{NJL}_{2}$ model
}

\author{
T. G. Khunjua, ${ }^{1}$ K. G. Klimenko, ${ }^{2}$ and R. N. Zhokhov ${ }^{3}$ \\ ${ }^{1}$ Faculty of Physics, Moscow State University, 119991, Moscow, Russia \\ ${ }^{2}$ State Research Center of Russian Federation-Institute for High Energy Physics, \\ NRC "Kurchatov Institute", 142281, Protvino, Moscow Region, Russia \\ ${ }^{3}$ Pushkov Institute of Terrestrial Magnetism, Ionosphere and Radiowave Propagation (IZMIRAN), \\ 108840 Troitsk, Moscow, Russia
}

(Received 12 May 2019; published 9 August 2019)

\begin{abstract}
In this paper we investigate in the large- $N_{c}$ limit ( $N_{c}$ is the number of colored quarks) the phase structure of a massless $(1+1)$-dimensional quark model with four-quark interaction and in the presence of baryon $\left(\mu_{B}\right)$, isospin $\left(\mu_{I}\right)$ and chiral isospin $\left(\mu_{I 5}\right)$ chemical potentials as well as at nonzero temperature. It is established that the chiral isospin chemical potential leads to the generation of charged pion condensation (PC) in dense (nonzero baryon density) and chiral asymmetric quark matter for a wide range of isospin densities. It is shown that there exists a duality correspondence between the chiral symmetry breaking and the charged PC phenomena at any values of temperature even for very hot quark gluon plasma. Moreover, it is shown that a charged PC phase with nonzero baryon density can be induced in the model at comparatively high temperatures. This opens up new possible physical systems, where it can be of importance, such as heavy-ion collisions, new-born neutron stars (proto-neutron stars), supernovas as well as neutron star mergers.
\end{abstract}

DOI: $10.1103 /$ PhysRevD.100.034009

\section{INTRODUCTION}

Recently, much attention has been paid to the study of dense baryon (quark) media with isotopic (isospin) asymmetry (different densities of $u$ and $d$ quarks). Such matter can exist inside compact stars, it can appear in heavy-ion collision experiments, etc., and it is usually described in terms of different nonperturbative QCD methods or effective QCD-like theories such as chiral effective Lagrangians or, especially, Nambu-Jona-Lasinio (NJL) type models [1] with nonzero baryon $\mu_{B}$ and isospin $\mu_{I}$ chemical potentials. It turns out that the $\left(\mu_{I}, \mu_{B}\right)$ phase diagram of these models gives us the opportunity to better understand phenomena such as chiral symmetry restoration [2-6], color superconductivity [7-9], and the charged pion condensation (PC) effect [10-22] (let us also recall ideas about pion stars [23]) that can be observed in dense quark matter with isotopic asymmetry.

While the reality of the first two of the above phenomena is beyond doubt, the possibility of condensation of charged pions in a dense quark medium has not been reliably

Published by the American Physical Society under the terms of the Creative Commons Attribution 4.0 International license. Further distribution of this work must maintain attribution to the author(s) and the published article's title, journal citation, and DOI. Funded by SCOAP ${ }^{3}$. established (at least in the framework of the NJL model considerations). Indeed, for some values of the model parameters (the coupling constant $G$, the cutoff parameter $\Lambda$, etc.), the phase of quark matter with nonzero baryon density, in which the charged pions are condensed, is allowed by the NJL models. However, this effect is prohibited by the NJL models for other physically interesting $G$ and $\Lambda$ values [12]. In addition, if the condition of electric neutrality is imposed on quark matter, then the charged PC phenomenon strongly depends on the size of the bare quark mass $m_{0}$. In particular, it turns out that in the framework of the NJL models the condensation of charged pions is forbidden in a medium with nonzero baryon density if $m_{0}$ reaches physically acceptable values of 5-10 MeV (see in Ref. [15]). However, on the basis of the toy $(1+1)$-dimensional model with baryon and isospin chemical potentials, it was shown in Refs. [16,17] that there are factors (the spatial sizes of the system or the spatial inhomogeneity of its condensates) that can stimulate the appearance of the charged PC phenomenon in dense quark matter.

Quite recently, it was found that in dense quark medium in the presence of an external magnetic field two other effects, chiral magnetic and chiral separation effects [24,25], can be observed. Thus they can be realized in compact stars and heavy-ion collision experiments, etc. Usually, these phenomena are inherent in such environments in which there is a 
chiral imbalance (or asymmetry), i.e. when in dense quark matter there is a difference between the density $n_{R}$ of all right-handed quarks and the density $n_{L}$ of all left-handed quarks. The quantity $n_{5} \equiv n_{R}-n_{L}$ is called the chiral density of the system. It has been argued that the chiral density $n_{5}$ can be generated dynamically at high temperatures, for example, in the fireball after heavy-ion collisions, by virtue of the Adler-Bell-Jackiw anomaly and quarks interacting with gauge (gluon) field configurations with nontrivial topology, named sphalerons. In the presence of an external strong magnetic field, which can be produced in heavy-ion collisions as well, this can lead to the so-called chiral magnetic effect [24]. Moreover, in the presence of an external magnetic field the chiral density $n_{5}$ can be produced (even at a rather low temperature) in dense quark matter due to the so-called chiral separation effect [25] (it can also be produced under fast rotations of the system due to the socalled chiral vortical effect). Now let us notice that usually when one talks about the chiral density $n_{5}$ one implies that the chiral density $n_{u 5}$ of $u$ quarks and chiral density $n_{d 5}$ of $d$ quarks are equal to each other (it is evident that $\left.n_{5}=n_{5 u}+n_{5 d}\right)$. Indeed, that is the case when one has in mind the mechanism of generation of chiral imbalance at high temperatures due to the nontrivial topology of the gauge-field configuration. In this case it is quite plausible that $n_{u 5}=n_{d 5}$ due to the fact that the gluon field interacts with different quark flavors in exactly the same way and does not feel the difference between flavors. But another mechanism, the chiral separation effect, is sensitive to the flavor of quarks (as it was shown in Appendix A in Refs. [26,27]). So in dense quark matter $\left(\mu_{B} \neq 0\right)$ a strong magnetic field separates $u$ and $d$ quarks in different ways. As a result, we see that, e.g., in such astrophysical objects as magnetars there might exist areas, in which the quantity $n_{I 5}=$ $n_{5 u}-n_{5 d}$, called the chiral isospin density, is not zero. Moreover, it has been argued that chiral imbalance is generated by parallel magnetic and electric fields [28], and one can generalize these arguments to chiral isospin imbalance as well.

So in the most general case, chiral imbalance is described by two chemical potentials - the chiral $\mu_{5}$ and chiral isospin (or isotopic) $\mu_{I 5}$ chemical potentials-which are thermodynamically conjugated to $n_{5}$ and $n_{I 5}$, respectively. The first, $\mu_{5}$, is usually used when isotopic asymmetry of quark matter is absent, i.e. in the case $\mu_{I}=0[29,30]$. The second, $\mu_{I 5}$, might be taken into account when, in addition to chiral, there is also isotopic asymmetry of matter, in which the charged PC phenomenon may occur, etc. [31]. In particular, it was established in the framework of NJL models that $\mu_{5}$ catalyzes the chiral symmetry breaking (CSB) [30,31], whereas $\mu_{I 5}$ promotes the charged PC in dense quark matter [31].

It was also shown in Ref. [31] that in the leading order of the $1 / N_{c}$ expansion the most general $\left(\mu_{B}, \mu_{I}, \mu_{5}, \mu_{I 5}\right)$-phase portrait of the massless NJL model is symmetric with respect to a so-called dual transformation between charged $\mathrm{PC}$ and CSB phases. If the bare quark mass $m_{0}$ is not zero, then this duality is only an approximate, although quite accurate, symmetry of the NJL model phase portrait (see Ref. [26]). In addition, it was established in this paper that the chiral isospin chemical potential $\mu_{I 5}$ promotes the charged PC phenomenon in dense quark matter even at physically acceptable values of $m_{0}$.

One of the drawbacks of the $(3+1)$-dimensional NJL $\left(\mathrm{NJL}_{4}\right)$ model is its nonrenormalizability. Therefore, the predictions of this effective model depend on the cutoff parameter, which is typically chosen to be of the order of $1 \mathrm{GeV}$. So, the results when using the $\mathrm{NJL}_{4}$ model are valid only at comparatively low energies, temperatures and densities (chemical potentials). However, there also exists a class of renormalizable theories - the $(1+1)$-dimensional chiral Gross-Neveu (GN)-type models $[32,33]^{1}$ — that can be used as a laboratory for the qualitative simulation of specific properties of QCD at arbitrary energies. Renormalizability, asymptotic freedom, as well as the spontaneous chiral symmetry breaking (in vacuum) are the most fundamental inherent features both for QCD and all $\mathrm{NJL}_{2}$-type models. In addition, the $\mu_{B}-T$ phase diagram (where $T$ denotes temperature) is qualitatively the same for both the QCD and $\mathrm{NJL}_{2}$ models [34-37]. Let us further mention that $(1+1)$-dimensional Gross-Neveutype models are also suitable for the description of physics in quasi-one-dimensional condensed matter systems like polyacetylene [38]. It is currently well understood (see, e.g., the discussion in Refs. $[36,37,39]$ ) that the usual no-go theorem [40], which generally forbids the spontaneous breaking of any continuous symmetry in two-dimensional spacetime, does not work in the limit $N_{c} \rightarrow \infty$, where $N_{c}$ is the number of colored quarks. This follows directly from the fact that in the limit of large $N_{c}$ the quantum fluctuations, which would otherwise destroy the long-range order corresponding to a spontaneous symmetry breaking, are suppressed by $1 / N_{c}$ factors. Thus, the effects inherent in real dense quark matter, such as the CSB phenomenon [spontaneous breaking of the continuous axial $U(1)$ symmetry] or charged pion condensation (spontaneous breaking of the continuous isospin symmetry) might be simulated in terms of simpler $\mathrm{NJL}_{2}$-type models, though only in the leading order of the large- $N_{c}$ approximation (see, e.g., Refs. [39,41-45], respectively).

This paper is devoted to the investigation of such phenomena of dense quark matter as CSB and charged PC, as well as their mutual influence on each other, in the framework of an extended toy $\mathrm{NJL}_{2}$ model with two massless quark flavors and in the presence of the baryon

\footnotetext{
${ }^{1}$ Below we shall use the notation " $\mathrm{NJL}_{2}$ model" instead of "chiral GN model" for $(1+1)$-dimensional models with continuous chiral and/or isotopic, etc., symmetries, since the chiral structure of their Lagrangians is the same as that of the corresponding $(3+1)$-dimensional NJL models.
} 
$\mu_{B}$, isospin $\mu_{I}$ as well as chiral isospin $\mu_{I 5}$ chemical potentials (for simplicity, we only consider the case $\mu_{5}=0$ ). Of course, in this case, in two dimensions, a significant portion of the physical processes that can occur in a real dense quark medium in $(3+1)$ dimensions, is removed from our consideration. However, as discussed, e.g., in Ref. [46], the dynamics of the pairing of fermions in strong magnetic fields is $(1+1)$ dimensional. Therefore, phenomena of real dense quark matter such as the spontaneous breaking of chiral symmetry, the condensation of charged pions and other pairing phenomena in strong magnetic fields can be investigated effectively in the framework of two-dimensional models. The chemical potential $\mu_{I 5}$ is also included in our consideration, because the regions with $\mu_{I 5} \neq 0$ can appear (due to the chiral separation effect) in neutron stars or arise in quark matter formed by the collision of heavy ions just under the influence of a strong magnetic field (this fact was discussed in Appendix A of Refs. [26,27]). Moreover, in order to avoid the no-go theorem [40], we perform all calculations in the leading order of the large- $N_{c}$ technique. To clarify the true role of the chiral isospin $\mu_{I 5}$ chemical potential in the creation of the CSB and charged PC in dense quark matter, it is supposed throughout the paper that all condensates are spatially homogeneous. ${ }^{2}$ Under this constraint the model was already investigated in Refs. [41,42] at $\mu_{I 5}=0$, where it was shown that the charged PC phase with nonzero baryon density is forbidden at arbitrary values of $\mu_{B}$ and $\mu_{I}$ (at zero chiral imbalance). In contrast, we show that at $\mu_{I 5} \neq 0$, i.e. when there is an isotopic chiral imbalance of the system, the charged PC phase with nonzero baryon density is allowed to exist for a wide range of isospin densities.

In addition, we show that in the leading order of the large- $N_{c}$ approximation there arises a duality between CSB and charged PC phenomena in the framework of the $\mathrm{NJL}_{2}$ model under consideration for the case of cold quark matter as well as for hot media. It means that if at $\mu_{I}=A$ and $\mu_{I 5}=B$ (at arbitrary fixed chemical potential $\mu_{B}$ ), e.g., the CSB (or the charged PC) phase is realized in the model, then at the permuted values of these chemical potentials, i.e. at $\mu_{I}=B$ and $\mu_{I 5}=A$, the charged PC (or the CSB) phase is arranged. So, it is enough to know the phase structure of the model at $\mu_{I}<\mu_{I 5}$, in order to establish the phase structure at $\mu_{I}>\mu_{I 5}$ and vice versa. Knowing condensates and other dynamical and thermodynamical quantities of the system, e.g. in the CSB phase, one can then obtain the corresponding quantities in the dually conjugated charged PC phase of the model, by simply performing there the duality transformation, $\mu_{I} \leftrightarrow \mu_{I 5} .{ }^{3}$ Earlier, similar dualities between the charged PC and

\footnotetext{
${ }^{2}$ As it was noted above, the spatial inhomogeneity of condensates by itself can cause charged PC in dense baryon matter [17].
}

chiral symmetry breaking phenomena were observed in the framework of the orbifold equivalence formalism in the limit of large $N_{c}$ [49]. Namely, it was shown that the whole phase diagram of QCD at the chiral chemical potential must be identical to that of QCD at the isotopic chemical potential in the chiral limit, where the charged pion condensation is replaced by the chiral condensation.

Note that the phase structure of the $\mathrm{NJL}_{2}$ model under consideration with nonzero values of $\mu_{B}, \mu_{I}$, and $\mu_{I 5}$ has already been studied in Ref. [50] at $T=0$, but an elusive error was made there. Formally, in Ref. [50] all calculations, both numerical and analytical, are correct but one of the statements in the Appendix, which only at first glance may seem to be correct, turned out to be untrue, and this led to an incorrect expression for the thermodynamic potential of the model (see below). However, it is interesting that although the basic qualitative conclusions are still true, some important details of the phase portrait have changed. The predictions became stronger and more interesting for the application to real physical scenarios. This paper is devoted to the investigation and the analysis of these changes, and we consider the influence of the temperature on the phase diagram and the duality of the model in this case.

The paper is organized as follows. In Sec. II a toy $(1+1)$-dimensional massless NJL-type model with two quark flavors ( $u$ and $d$ quarks) and with three kinds of chemical potentials, $\mu_{B}, \mu_{I}, \mu_{I 5}$, is presented. Next, we discuss the symmetries of the model under consideration and the unrenormalized expression for the thermodynamic potential (TDP) for the cases of both zero and nonzero temperature is obtained in the leading order of the large- $N_{c}$ expansion. Here the dual symmetry of the model TDP is established at $T \geq 0$. Section III is devoted to the calculation of the TDP at $T=0$ at both zero and nonzero values of the chemical potentials. Then, in Sec. IV, the phase structure of the model is investigated at $T=0$. In this section, on the basis of several phase diagrams, we demonstrate the existence of a duality between CSB and charged PC phenomena as well the fact that $\mu_{I 5}$ promotes the realization of the charged PC phase in dense quark matter. It means that the charged PC phase with nonzero baryon density can be realized in this model only at $\mu_{I 5}>0$. Similar results are obtained in Sec. V, where the case of nonzero temperature is considered. In Sec. VI the main results and conclusions of the paper are formulated, and we emphasize the similarity of the phase properties of the NJL model in two and four spacetime dimensions with isotopic chiral asymmetry. Some technical details are relegated to Appendices A and B.

\footnotetext{
${ }^{3}$ Note that another kind of duality correspondence, the duality between CSB and superconductivity, was demonstrated both in $(1+1)$ - and $(2+1)$-dimensional NJL models $[47,48]$.
} 


\section{THE MODEL AND ITS THERMODYNAMIC POTENTIAL}

\section{A. The zero-temperature case}

We consider in the chiral limit a $(1+1)$-dimensional NJL model in order to mimic the phase structure of real dense quark matter with two massless quark flavors ( $u$ and $d$ quarks). Its Lagrangian, which is symmetrical under the global color $\mathrm{SU}\left(N_{c}\right)$ group, has the form

$$
\begin{aligned}
L= & \bar{q}\left[\gamma^{\nu} \mathrm{i} \partial_{\nu}+\frac{\mu_{B}}{3} \gamma^{0}+\frac{\mu_{I}}{2} \tau_{3} \gamma^{0}+\frac{\mu_{I 5}}{2} \tau_{3} \gamma^{0} \gamma^{5}\right] q \\
& +\frac{G}{N_{c}}\left[(\bar{q} q)^{2}+\left(\bar{q} \mathrm{i} \gamma^{5} \vec{\tau} q\right)^{2}\right],
\end{aligned}
$$

where the quark field $q(x) \equiv q_{i \alpha}(x)$ is a flavor doublet $(i=1,2$ or $i=u, d)$ and color $N_{c}$-plet $\left(\alpha=1, \ldots, N_{c}\right)$ as well as a two-component Dirac spinor [the summation in Eq. (1) over flavor, color, and spinor indices is implied]; $\tau_{k}$ $(k=1,2,3)$ are Pauli matrices in two-dimensional flavor space. The Dirac $\gamma^{\nu}$ matrices $(\nu=0,1)$ and $\gamma^{5}$ in Eq. (1) are matrices in two-dimensional spinor space,

$$
\begin{aligned}
& \gamma^{0}=\left(\begin{array}{ll}
0 & 1 \\
1 & 0
\end{array}\right), \quad \gamma^{1}=\left(\begin{array}{cc}
0 & -1 \\
1 & 0
\end{array}\right), \\
& \gamma^{5}=\gamma^{0} \gamma^{1}=\left(\begin{array}{cc}
1 & 0 \\
0 & -1
\end{array}\right) .
\end{aligned}
$$

Note that at $\mu_{I 5}=0$ the model was already investigated in detail, e.g., in Refs [41-43,45]. It is evident that the model (1) is a generalization of the two-dimensional GN model [32] with a single massless quark color $N_{c}$-plet to the case of two quark flavors and additional baryon $\mu_{B}$, isospin $\mu_{I}$ and axial isospin $\mu_{I 5}$ chemical potentials. These parameters are introduced in order to describe in the framework of the model (1) quark matter with nonzero baryon $n_{B}$, isospin $n_{I}$ and axial isospin $n_{I 5}$ densities, respectively. It is evident that the Lagrangian (1), both at $\mu_{I 5}=0$ and $\mu_{I 5} \neq 0$, is invariant with respect to the Abelian $U_{B}(1), U_{I_{3}}(1)$ and $U_{A I_{3}}(1)$ groups, where ${ }^{4}$

$$
\begin{aligned}
U_{B}(1): q & \rightarrow \exp (\mathrm{i} \alpha / 3) q ; \quad U_{I_{3}}(1): q \rightarrow \exp \left(\mathrm{i} \beta \tau_{3} / 2\right) q ; \\
U_{A I_{3}}(1): q & \rightarrow \exp \left(\mathrm{i} \omega \gamma^{5} \tau_{3} / 2\right) q .
\end{aligned}
$$

[In Eq. (3) the real parameters $\alpha, \beta, \omega$ specify an arbitrary element of the $U_{B}(1), U_{I_{3}}(1)$ and $U_{A I_{3}}(1)$ groups, respectively.] So the quark bilinears $\frac{1}{3} \bar{q} \gamma^{0} q, \frac{1}{2} \bar{q} \gamma^{0} \tau^{3} q$ and $\frac{1}{2} \bar{q} \gamma^{0} \gamma^{5} \tau^{3} q$ are the zero components of corresponding conserved currents. Their ground-state expectation values are just the baryon $n_{B}$, isospin $n_{I}$ and chiral (axial) isospin

\footnotetext{
${ }^{4}$ Recall for the following that $\exp \left(\mathrm{i} \beta \tau_{3} / 2\right)=\cos (\beta / 2)+$ $\mathrm{i} \tau_{3} \sin (\beta / 2), \exp \left(\mathrm{i} \omega \gamma^{5} \tau_{3} / 2\right)=\cos (\omega / 2)+\mathrm{i} \gamma^{5} \tau_{3} \sin (\omega / 2)$.
}

$n_{I 5}$ densities of quark matter, i.e. $n_{B}=\frac{1}{3}\left\langle\bar{q} \gamma^{0} q\right\rangle, n_{I}=$ $\frac{1}{2}\left\langle\bar{q} \gamma^{0} \tau^{3} q\right\rangle$ and $n_{I 5}=\frac{1}{2}\left\langle\bar{q} \gamma^{0} \gamma^{5} \tau^{3} q\right\rangle$. As usual, the quantities $n_{B}, n_{I}$ and $n_{I 5}$ can also be found by differentiating the thermodynamic potential of the system with respect to the corresponding chemical potentials. The goal of the present paper is to investigate the ground-state properties and phase structure of the system (1) and its dependence on the chemical potentials $\mu_{B}, \mu_{I}$ and $\mu_{I 5}$.

To find the thermodynamic potential of the system, we use a semibosonized version of the Lagrangian (1), which contains composite bosonic fields $\sigma(x)$ and $\pi_{a}(x)$ ( $a=1$, 2,3 ) (in what follows, we use the notations $\mu \equiv \mu_{B} / 3, \nu \equiv$ $\mu_{I} / 2$ and $\nu_{5} \equiv \mu_{I 5} / 2$ ):

$$
\begin{aligned}
\tilde{L}= & \bar{q}\left[\gamma^{\rho} \mathrm{i} \partial_{\rho}+\mu \gamma^{0}+\nu \tau_{3} \gamma^{0}+\nu_{5} \tau_{3} \gamma^{0} \gamma^{5}-\sigma-\mathrm{i} \gamma^{5} \pi_{a} \tau_{a}\right] q \\
& -\frac{N_{c}}{4 G}\left[\sigma \sigma+\pi_{a} \pi_{a}\right] .
\end{aligned}
$$

In Eq. (4) the summation over repeated indices is implied. From the Lagrangian (4) one gets the Euler-Lagrange equations for the bosonic fields

$\sigma(x)=-2 \frac{G}{N_{c}}(\bar{q} q), \quad \pi_{a}(x)=-2 \frac{G}{N_{c}}\left(\bar{q} \mathrm{i} \gamma^{5} \tau_{a} q\right)$.

Note that the composite bosonic field $\pi_{3}(x)$ can be identified with the physical $\pi_{0}$ meson, whereas the physical $\pi^{ \pm}(x)$-meson fields are the following combinations of the composite fields: $\pi^{ \pm}(x)=\left(\pi_{1}(x) \mp i \pi_{2}(x)\right) /$ $\sqrt{2}$. Obviously, the semibosonized Lagrangian $\tilde{L}$ is equivalent to the initial Lagrangian (1) when using the equations (5). Furthermore, it is clear from Eqs. (3) and (5) and footnote 4 that the bosonic fields transform under the isospin $U_{I_{3}}(1)$ and axial isospin $U_{A I_{3}}(1)$ groups in the following manner:

$$
\begin{aligned}
& U_{I_{3}}(1): \sigma \rightarrow \sigma ; \pi_{3} \rightarrow \pi_{3} ; \pi_{1} \rightarrow \cos (\beta) \pi_{1} \\
&+\sin (\beta) \pi_{2} ; \pi_{2} \rightarrow \cos (\beta) \pi_{2}-\sin (\beta) \pi_{1}, \\
& U_{A I_{3}}(1): \pi_{1} \rightarrow \pi_{1} ; \pi_{2} \rightarrow \pi_{2} ; \sigma \rightarrow \cos (\omega) \sigma \\
& \quad+\sin (\omega) \pi_{3} ; \pi_{3} \rightarrow \cos (\omega) \pi_{3}-\sin (\omega) \sigma .
\end{aligned}
$$

In general the phase structure of a given model is characterized by the behavior of some quantities, called order parameters (or condensates), vs external conditions (temperature, chemical potentials, etc.). In the case of model (1), such order parameters are the ground-state expectation values of the composite fields, i.e. the quantities $\langle\sigma(x)\rangle$ and $\left\langle\pi_{a}(x)\right\rangle(a=1,2,3)$. It is clear from Eq. (6) that if $\langle\sigma(x)\rangle \neq 0$ and/or $\left\langle\pi_{3}(x)\right\rangle \neq 0$, then the axial isospin $U_{A I_{3}}(1)$ symmetry of the model is spontaneously broken, whereas at $\left\langle\pi_{1}(x)\right\rangle \neq 0$ and/or $\left\langle\pi_{2}(x)\right\rangle \neq 0$ we have a spontaneous breaking of the isospin $U_{I_{3}}(1)$ symmetry. Since in the last case the ground-state expectation values 
(condensates) of both fields $\pi^{+}(x)$ and $\pi^{-}(x)$ are not zero, this phase is usually called the charged pion condensation (PC) phase. The ground-state expectation values $\langle\sigma(x)\rangle$ and $\left\langle\pi_{a}(x)\right\rangle$ are the coordinates of the global minimum point of the thermodynamic potential $\Omega\left(\sigma, \pi_{a}\right)$ of the system.

Starting from the linearized semibosonized model Lagrangian (4), one obtains in the leading order of the large- $N_{c}$ expansion (i.e. in the one-fermion-loop approximation) the following path integral expression for the effective action $\mathcal{S}_{\text {eff }}\left(\sigma, \pi_{a}\right)$ of the bosonic $\sigma(x)$ and $\pi_{a}(x)$ fields:

$$
\exp \left(\mathrm{i} \mathcal{S}_{\mathrm{eff}}\left(\sigma, \pi_{a}\right)\right)=N^{\prime} \int[d \bar{q}][d q] \exp \left(\mathrm{i} \int \tilde{L} d^{2} x\right),
$$

where

$$
\mathcal{S}_{\text {eff }}\left(\sigma, \pi_{a}\right)=-N_{c} \int d^{2} x\left[\frac{\sigma^{2}+\pi_{a}^{2}}{4 G}\right]+\tilde{\mathcal{S}}_{\text {eff }},
$$

and $N^{\prime}$ is a normalization constant. The quark contribution to the effective action, i.e. the term $\tilde{\mathcal{S}}_{\text {eff }}$ in Eq. (7), is given by

$$
\begin{aligned}
\exp \left(\tilde{\mathrm{i}}_{\text {eff }}\right) & =N^{\prime} \int[d \bar{q}][d q] \exp \left(\mathrm{i} \int[\bar{q} \mathrm{D} q] d^{4} x\right) \\
& =[\operatorname{Det} D]^{N_{c}} .
\end{aligned}
$$

In Eq. (8) we have used the notation $\mathrm{D} \equiv D \times \mathrm{I}_{c}$, where $\mathrm{I}_{c}$ is the unit operator in the $N_{c}$-dimensional color space and

$D \equiv \gamma^{\rho} \mathrm{i} \partial_{\rho}+\mu \gamma^{0}+\nu \tau_{3} \gamma^{0}+\nu_{5} \tau_{3} \gamma^{0} \gamma^{5}-\sigma-\mathrm{i} \gamma^{5} \pi_{a} \tau_{a}$

is the Dirac operator, which acts in the flavor, spinor as well as coordinate spaces only. Using the general formula Det $D=\exp \operatorname{Tr} \ln D$, one obtains for the effective action the following expression:
$\mathcal{S}_{\text {eff }}\left(\sigma, \pi_{a}\right)=-N_{c} \int d^{2} x\left[\frac{\sigma^{2}+\pi_{a}^{2}}{4 G}\right]-\mathrm{i} N_{c} \operatorname{Tr}_{s f x} \ln D$,

where the $\operatorname{Tr}$ operation stands for the trace in spinor $(s)$, flavor $(f)$ as well as two-dimensional coordinate $(x)$ spaces, respectively. Using Eq. (10), we obtain the TDP $\Omega\left(\sigma, \pi_{a}\right)$ of the system:

$$
\begin{aligned}
\Omega\left(\sigma, \pi_{a}\right) & \equiv-\left.\frac{\mathcal{S}_{\text {eff }}\left(\sigma, \pi_{a}\right)}{N_{c} \int d^{2} x}\right|_{\sigma, \pi_{a}=\text { const }}=\frac{\sigma^{2}+\pi_{a}^{2}}{4 G}+\mathrm{i} \frac{\operatorname{Tr}_{s f x} \ln D}{\int d^{2} x} \\
& =\frac{\sigma^{2}+\pi_{a}^{2}}{4 G}+\mathrm{i} \operatorname{Tr}_{s f} \int \frac{d^{2} p}{(2 \pi)^{2}} \ln \bar{D}(p)
\end{aligned}
$$

where the $\sigma$ and $\pi_{a}$ fields are now $x$-independent quantities, and

$\bar{D}(p)=\not p+\mu \gamma^{0}+\nu \tau_{3} \gamma^{0}+\nu_{5} \tau_{3} \gamma^{0} \gamma^{5}-\sigma-\mathrm{i} \gamma^{5} \pi_{a} \tau_{a}$

is the momentum-space representation of the Dirac operator $D$ [Eq. (9)]. In what follows we are going to investigate the $\mu, \nu, \nu_{5}$ dependence of the global minimum point of the function $\Omega\left(\sigma, \pi_{a}\right)$ vs $\sigma, \pi_{a}$. To simplify the task, let us note that due to the $U_{I_{3}}(1) \times U_{A I_{3}}(1)$ invariance of the model, the TDP (11) depends effectively only on the two combinations $\sigma^{2}+\pi_{3}^{2}$ and $\pi_{1}^{2}+\pi_{2}^{2}$ of the bosonic fields, as is easily seen from Eq. (6). In this case, without loss of generality, one can put $\pi_{2}=\pi_{3}=0$ in Eq. (11), and study the TDP (11) as a function of only two variables: $M \equiv \sigma$ and $\Delta \equiv \pi_{1}$. Taking into account this constraint in Eqs. (12) and (11) as well as the general relation

$$
\operatorname{Tr}_{s f} \ln \bar{D}(p)=\ln \operatorname{Det} \bar{D}(p)=\sum_{i} \ln \epsilon_{i}
$$

where the summation over all four eigenvalues $\epsilon_{i}$ of the $4 \times 4$ matrix $\bar{D}(p)$ is implied and

$$
\epsilon_{1,2,3,4}=-M \pm \sqrt{\left(p_{0}+\mu\right)^{2}-p_{1}^{2}-\Delta^{2}+\nu^{2}-\nu_{5}^{2} \pm 2 \sqrt{\left[\left(p_{0}+\mu\right) \nu+p_{1} \nu_{5}\right]^{2}-\Delta^{2}\left(\nu^{2}-\nu_{5}^{2}\right)}},
$$

we have from Eq. (11)

$$
\Omega(M, \Delta)=\frac{M^{2}+\Delta^{2}}{4 G}+\mathrm{i} \int \frac{d^{2} p}{(2 \pi)^{2}} \ln P_{4}\left(p_{0}\right) .
$$

In Eq. (15) we use the notation

$$
P_{4}\left(p_{0}\right)=\epsilon_{1} \epsilon_{2} \epsilon_{3} \epsilon_{4}=\eta^{4}-2 a \eta^{2}-b \eta+c,
$$

where $\eta=p_{0}+\mu$ and

$$
\begin{aligned}
& a=M^{2}+\Delta^{2}+p_{1}^{2}+\nu^{2}+\nu_{5}^{2}, \quad b=8 p_{1} \nu \nu_{5}, \\
& c=a^{2}-4 p_{1}^{2}\left(\nu^{2}+\nu_{5}^{2}\right)-4 M^{2} \nu^{2}-4 \Delta^{2} \nu_{5}^{2}-4 \nu^{2} \nu_{5}^{2} .
\end{aligned}
$$

Thus, it follows from Eqs. (16) and (17) that the TDP (15) is invariant with respect to the so-called duality transformation (for an analogous case of duality between chiral and superconducting condensates, see Refs. $[47,48]$ ),

$$
\mathcal{D}: M \leftrightarrow \Delta, \nu \leftrightarrow \nu_{5} .
$$


In powers of $\Delta$ and $M$ the fourth-degree polynomial $P_{4}\left(p_{0}\right)$ has the following forms:

$$
\begin{aligned}
P_{4}\left(p_{0}\right) \equiv & \Delta^{4}-2 \Delta^{2}\left(\eta^{2}-p_{1}^{2}-M^{2}+\nu_{5}^{2}-\nu^{2}\right) \\
& +\left[M^{2}+\left(p_{1}-\nu_{5}\right)^{2}-(\eta+\nu)^{2}\right] \\
& \times\left[M^{2}+\left(p_{1}+\nu_{5}\right)^{2}-(\eta-\nu)^{2}\right] \\
\equiv & M^{4}-2 M^{2}\left(\eta^{2}-p_{1}^{2}-\Delta^{2}+\nu^{2}-\nu_{5}^{2}\right) \\
& +\left[\Delta^{2}+\left(p_{1}-\nu\right)^{2}-\left(\eta+\nu_{5}\right)^{2}\right]\left[\Delta^{2}\right. \\
& \left.+\left(p_{1}+\nu\right)^{2}-\left(\eta-\nu_{5}\right)^{2}\right] .
\end{aligned}
$$

Note that according to the general theorem of algebra, the polynomial $P_{4}\left(p_{0}\right)$ can also be presented in the form

$$
P_{4}\left(p_{0}\right) \equiv\left(p_{0}-p_{01}\right)\left(p_{0}-p_{02}\right)\left(p_{0}-p_{03}\right)\left(p_{0}-p_{04}\right),
$$

where the roots $p_{01}, p_{02}, p_{03}$ and $p_{04}$ of this polynomial are the energies of quasiparticle or quasiantiparticle excitations of the system. In particular, it follows from Eq. (19) that at $\Delta=0$ the set of roots $p_{0 i}$ looks like

$$
\begin{aligned}
\left.\left\{p_{01}, p_{02}, p_{03}, p_{04}\right\}\right|_{\Delta=0}= & \left\{-\mu-\nu \pm \sqrt{M^{2}+\left(p_{1}-\nu_{5}\right)^{2}},\right. \\
& \left.-\mu+\nu \pm \sqrt{M^{2}+\left(p_{1}+\nu_{5}\right)^{2}}\right\},
\end{aligned}
$$

whereas it is clear from Eq. (20) that at $M=0$ it has the form

$$
\begin{aligned}
\left.\left\{p_{01}, p_{02}, p_{03}, p_{04}\right\}\right|_{M=0}= & \left\{-\mu-\nu_{5} \pm \sqrt{\Delta^{2}+\left(p_{1}-\nu\right)^{2}},\right. \\
& \left.-\mu+\nu_{5} \pm \sqrt{\Delta^{2}+\left(p_{1}+\nu\right)^{2}}\right\}
\end{aligned}
$$

Taking into account the relation (21) as well as the formula (it was proved, e.g., in Ref. [17])

$$
\int_{-\infty}^{\infty} d p_{0} \ln \left(p_{0}-K\right)=\mathrm{i} \pi|K|
$$

(being true up to an infinite term independent on the real quantity $K$ ), it is possible to integrate in Eq. (15) over $p_{0}$. Then, the unrenormalized TDP (15) can be presented in the following form:

$$
\begin{aligned}
\Omega(M, \Delta) \equiv & \Omega^{u n}(M, \Delta)=\frac{M^{2}+\Delta^{2}}{4 G} \\
& -\int_{-\infty}^{\infty} \frac{d p_{1}}{4 \pi}\left(\left|p_{01}\right|+\left|p_{02}\right|+\left|p_{03}\right|+\left|p_{04}\right|\right) .
\end{aligned}
$$

It is clear directly from the relations (16) and (17) that the TDP (15) is an even function over each of the variables $M$ and $\Delta$. In addition, it is invariant under each of the transformations $\mu \rightarrow-\mu, \nu \rightarrow-\nu, \nu_{5} \rightarrow-\nu_{5} .{ }^{5}$ Hence, without loss of generality in the following we only consider the values $\mu \geq 0, \nu \geq 0, \nu_{5} \geq 0, M \geq 0$, and $\Delta \geq 0$. Finally, note that the integrand in Eq. (25) is not an even function of $p_{1}$ (see also Appendix A).

\section{B. The nonzero-temperature case}

Although the effect of nonzero temperatures is quite predictable (one can expect that the temperature just restores all the broken symmetries of the model), here we consider nonzero temperatures because it is instructive to know how robust the charged PC phase is under temperature.

To introduce a finite temperature $T$, it is very convenient to use the zero-temperature expression (15) for the TDP,

$$
\begin{aligned}
\Omega(M, \Delta)= & \frac{M^{2}+\Delta^{2}}{4 G}+\mathrm{i} \int \frac{d^{2} p}{(2 \pi)^{2}} \ln \left(p_{0}-p_{01}\right) \\
& \times\left(p_{0}-p_{02}\right)\left(p_{0}-p_{03}\right)\left(p_{0}-p_{04}\right),
\end{aligned}
$$

where we took into account Eq. (21). Then, to find the temperature-dependent TDP $\Omega_{T}(M, \Delta)$ one should replace in Eq. (26) the integration over $p_{0}$ in favor of the summation over Matsubara frequencies $\omega_{n}$ by the rule

$$
\begin{aligned}
\int_{-\infty}^{\infty} \frac{d p_{0}}{2 \pi}(\cdots) & \rightarrow i T \sum_{n=-\infty}^{\infty}(\cdots), \\
p_{0} & \rightarrow p_{0 n} \equiv i \omega_{n} \equiv i \pi T(2 n+1), \\
n & =0, \pm 1, \pm 2, \ldots
\end{aligned}
$$

In the expression obtained, it is possible to sum over Matsubara frequencies using the general formula (the corresponding technique was presented, e.g., in Ref. [51])

$$
\begin{aligned}
\sum_{n=-\infty}^{\infty} \ln \left(i \omega_{n}-a\right) & =\ln [\exp (\beta|a| / 2)+\exp (-\beta|a| / 2)] \\
& =\frac{\beta|a|}{2}+\ln [1+\exp (-\beta|a|)],
\end{aligned}
$$

where $\beta=1 / T$. As a result, one can obtain the following expression for the TDP $\Omega_{T}(M, \Delta)$ :

\footnotetext{
${ }^{5}$ Indeed, if we perform simultaneously with $\mu \rightarrow-\mu$ the change of variables $p_{0} \rightarrow-p_{0}$ and $p_{1} \rightarrow-p_{1}$ in the integral (15), then one can easily see that the expression (15) remains intact. Finally, if only $\nu$ (only $\nu_{5}$ ) is replaced by $-\nu$ (by $-\nu_{5}$ ), we should transform $p_{1} \rightarrow-p_{1}$ in the integral (15) in order to see that the TDP remains unchanged.
} 
$\Omega_{T}(M, \Delta)=\Omega(M, \Delta)-T \sum_{i=1}^{4} \int_{-\infty}^{\infty} \frac{d p_{1}}{2 \pi} \ln \left(1+e^{-\beta\left|p_{0 i}\right|}\right)$,

where $\Omega(M, \Delta)$ is the TDP (25) of the system at zero temperature. Since each root $p_{0 i}$ in Eq. (29) is a dually $\mathcal{D}$-invariant quantity (see Appendix A), it is clear that the temperature-dependent TDP (29) is also symmetric with respect to the duality transformation $\mathcal{D}(18)$.

\section{CALCULATION OF THE TDP AT $T=0$}

\section{A. Thermodynamic potential in vacuum: The case of $\mu=0, \nu=0, \nu_{5}=0$}

First of all, let us obtain a finite, i.e. renormalized, expression for the TDP (25) at $\mu=0, \nu=0$ and $\nu_{5}=0$, i.e. in vacuum, and at zero temperature. Since in this case a thermodynamic potential is usually called an effective potential, we use for it the notation $V^{u n}(M, \Delta)$. As a consequence of Eqs. (15)-(17) and using Eq. (24), it is clear that at $\mu=\nu=\nu_{5}=0$ the effective potential $V^{u n}(M, \Delta)$ looks like

$$
\begin{aligned}
V^{u n}(M, \Delta) & =\frac{M^{2}+\Delta^{2}}{4 G}+2 i \int \frac{d^{2} p}{(2 \pi)^{2}} \ln \left[p_{0}^{2}-p_{1}^{2}-M^{2}-\Delta^{2}\right] \\
& =\frac{M^{2}+\Delta^{2}}{4 G}-\int_{-\infty}^{\infty} \frac{d p_{1}}{\pi} \sqrt{p_{1}^{2}+M^{2}+\Delta^{2}}
\end{aligned}
$$

It is evident that the effective potential (30) is an ultraviolet (UV)-divergent quantity. So, we need to renormalize it. This procedure consists of two steps. (i) First of all we need to regularize the divergent integral in Eq. (30), i.e. we suppose there that $\left|p_{1}\right|<\Lambda$. (ii) Second, we must suppose also that the bare coupling constant $G$ depends on the cutoff parameter $\Lambda$ in such a way that in the limit $\Lambda \rightarrow \infty$ one obtains a finite expression for the effective potential.

Following step (i) of this procedure, we have

$$
\begin{aligned}
V^{\mathrm{reg}}(M, \Delta)= & \frac{M^{2}+\Delta^{2}}{4 G}-\frac{2}{\pi} \int_{0}^{\Lambda} d p_{1} \sqrt{p_{1}^{2}+M^{2}+\Delta^{2}} \\
= & \frac{M^{2}+\Delta^{2}}{4 G}-\frac{1}{\pi}\left\{\Lambda \sqrt{\Lambda^{2}+M^{2}+\Delta^{2}}\right. \\
& \left.+\left(M^{2}+\Delta^{2}\right) \ln \frac{\Lambda+\sqrt{\Lambda^{2}+M^{2}+\Delta^{2}}}{\sqrt{M^{2}+\Delta^{2}}}\right\} .
\end{aligned}
$$

Further, according to step (ii) we suppose that in Eq. (31) the bare coupling $G \equiv G(\Lambda)$ has the following $\Lambda$ dependence:

$$
\frac{1}{4 G(\Lambda)}=\frac{1}{\pi} \ln \frac{2 \Lambda}{m}
$$

where $m$ is a new mass scale of the model, and $m$ is a free model parameter. It appears instead of the dimensionless bare coupling constant $G$ (dimensional transmutation) and, evidently, does not depend on a normalization point, i.e. it is a renormalization-invariant quantity. Substituting Eq. (32) into Eq. (31) and ignoring there an unessential term $\left(-\Lambda^{2} / \pi\right)$, we obtain in the limit $\Lambda \rightarrow \infty$ the finite and renormalization-invariant expression for the effective potential,

$$
V_{0}(M, \Delta)=\frac{M^{2}+\Delta^{2}}{2 \pi}\left[\ln \left(\frac{M^{2}+\Delta^{2}}{m^{2}}\right)-1\right] .
$$

\section{B. Calculation of the TDP (25) in the general case:$$
\boldsymbol{\mu}>\mathbf{0}, \nu>0, \nu_{5}>0
$$

In Appendix $A$ the properties of the quasiparticle energies $p_{0 i}$, where $i=1, \ldots, 4$, are investigated. In particular, it is clear from the asymptotic expansion (A12) that the integral over $p_{1}$ in Eq. (25) is ultraviolet divergent. It is possible to transform the expression (25) in the following way:

$$
\begin{aligned}
\Omega^{u n}(M, \Delta)= & \frac{M^{2}+\Delta^{2}}{4 G} \\
& -\left.\int_{-\infty}^{\infty} \frac{d p_{1}}{4 \pi}\left(\left|p_{01}\right|+\left|p_{02}\right|+\left|p_{03}\right|+\left|p_{04}\right|\right)\right|_{\mu=\nu=\nu_{5}=0} \\
& -\int_{-\infty}^{\infty} \frac{d p_{1}}{4 \pi}\left[\sum_{i=1}^{4}\left|p_{0 i}\right|-\left.\left(\sum_{i=1}^{4}\left|p_{0 i}\right|\right)\right|_{\mu=\nu=\nu_{5}=0}\right] .
\end{aligned}
$$

Since the asymptotic expansion (A12) does not depend on the chemical potentials $\mu, \nu$ and $\nu_{5}$, it is evident that the last integral in Eq. (34) is convergent and all UV divergences of the TDP are located in the first integral of Eq. (34). Moreover, it is clear due to the relation (A13) that the first two terms on the right-hand side of Eq. (34) are just the unrenormalized effective potential in vacuum (30). So to obtain a finite expression for the TDP (34), it is enough to proceed as in the previous subsection, where just these two terms, i.e. the vacuum effective potential, were renormalized. As a result, we have

$$
\begin{aligned}
\Omega^{\mathrm{ren}}(M, \Delta)= & V_{0}(M, \Delta) \\
& -\int_{-\infty}^{\infty} \frac{d p_{1}}{4 \pi}\left\{\left|p_{01}\right|+\left|p_{02}\right|+\left|p_{03}\right|+\left|p_{04}\right|\right. \\
& \left.-4 \sqrt{p_{1}^{2}+M^{2}+\Delta^{2}}\right\}
\end{aligned}
$$

where $V_{0}(M, \Delta)$ is the renormalized TDP (effective potential) (33) of the model at $\mu=\nu=\mu_{5}=0$. Moreover, we have used in Eq. (35) the relation (A13) for the sum of quasiparticle energies in vacuum. Note also that (as it 
follows from the considerations of Appendix A) the quasiparticle energies $p_{0 i}$, where $i=1, \ldots, 4$, are invariant with respect to the duality transformation (18). So the renormalized TDP (35) is also symmetric under the duality transformation $\mathcal{D}$.

In Ref. [50] the erroneous assumption was used that the quantity $\sum_{i=1}^{4}\left|p_{0 i}\right|$ is an even function of $p_{1}$. This further led to an incorrect expression for the TDP (35), in which the integration over $p_{1} \in(0,+\infty)$ was used, and to erroneous phase diagrams. Here we correct this mistake. Therefore, in Eq. (35), the integration over $p_{1} \in$ $(-\infty,+\infty)$ is carried out. However, the main qualitative conclusions about the properties of the chiral isospin chemical potential $\nu_{5}$ and the duality of the model under consideration do not change.

Let us denote by $\left(M_{0}, \Delta_{0}\right)$ the global minimum point (GMP) of the TDP (35). Then, by investigating the behavior of this point vs $\mu, \nu$ and $\nu_{5}$ it is possible to construct the $\left(\mu, \nu, \nu_{5}\right)$-phase portrait (diagram) of the model. A numerical algorithm for finding the quasi(anti) particle energies $p_{01}, p_{02}, p_{03}$, and $p_{04}$ is elaborated in Appendix A. Based on this algorithm, it is possible to study the TDP of the model in the region of sufficiently small values of chemical potentials, e.g., at $\mu, \nu, \nu_{5} \leq 2 m$, where we did not find any GMP of the form $\left(M_{0} \neq 0, \Delta_{0} \neq 0\right)$ (this conclusion probably will not change at larger values of chemical potentials). Strictly speaking, this property of the TDP is typical for the NJL models only in the chiral limit. At the physical point, i.e. when quarks have a nonzero bare mass, in the charged PC phase both the $\Delta_{0}$ and $M_{0}$ condensates are nonzero (see, e.g., Refs. [11,26,44]). But these condensates are of the same order of magnitude only in the transitory region (in the region between the CSB and charged PC phases), whereas deeper in the phases one of the condensates dominates. For example, in the charged PC phase, first (at $\nu$ slightly larger than half of the pion mass) there is a nonzero chiral condensate, or dynamical quark mass, $M_{0} \gg \Delta_{0}$ but it decreases very fast and at larger $\nu$ the chiral condensate $M_{0}$ is much smaller than the charged pion one, $\Delta_{0}$. Let us also recall that the chiral limit overall is a good approximation as it has been shown in Ref. [26]. Hence, in order to establish the phase portrait of the massless model (1), e.g., in the region $\mu, \nu, \nu_{5} \leq 2 m$, it is enough to study the projections $F_{1}(M) \equiv \Omega^{\text {ren }}(M, \Delta=0)$ and $F_{2}(\Delta) \equiv \Omega^{\text {ren }}(M=0, \Delta)$ of the TDP (35) onto the $M$ and $\Delta$ axes, correspondingly. Taking into account the relations (22) for the quasiparticle energies $p_{0 i}$ at $\Delta=0$, it is possible to obtain the following expression for the projection $F_{1}(M)$ :

$$
\begin{aligned}
F_{1}(M)= & \frac{M^{2}}{2 \pi} \ln \left(\frac{M^{2}}{m^{2}}\right)-\frac{M^{2}}{2 \pi}-\frac{\nu_{5}^{2}}{\pi} \\
& -\frac{\theta(|\mu-\nu|-M)}{2 \pi}\left(|\mu-\nu| \sqrt{(\mu-\nu)^{2}-M^{2}}-M^{2} \ln \frac{|\mu-\nu|+\sqrt{|\mu-\nu|^{2}-M^{2}}}{M}\right) \\
& -\frac{\theta(\mu+\nu-M)}{2 \pi}\left((\mu+\nu) \sqrt{(\mu+\nu)^{2}-M^{2}}-M^{2} \ln \frac{\mu+\nu+\sqrt{(\mu+\nu)^{2}-M^{2}}}{M}\right) .
\end{aligned}
$$

Then, the projection $F_{2}(\Delta)$ of the TDP onto the $\Delta$ axis can be obtained directly from Eq. (23) or, alternatively, using the dual symmetry $\mathcal{D}$ of the TDP,

$$
F_{2}(\Delta)=\left.F_{1}(\Delta)\right|_{\nu \leftrightarrow \nu_{5}}
$$

(Details of the derivation of these expressions are given in Appendix B.) After simple transformations, one can see that $F_{1}(M)$ and $F_{2}(\Delta)$ coincide at $\nu_{5}=0$ with the corresponding TDPs (12) and (13) of Ref. [43]. Moreover, it is obvious that the GMP of the TDP (35) is defined by a comparison between the minimum values of the functions $F_{1}(M)$ and $F_{2}(\Delta)$.

\section{Quark number density}

As it is clear from the above consideration, there are three phases in the model (1). The first one is the symmetric phase, which corresponds to the global minimum point
$\left(M_{0}, \Delta_{0}\right)$ of the TDP (35) of the form $\left(M_{0}=0, \Delta_{0}=0\right)$. In the CSB phase the TDP reaches its minimum value at the point $\left(M_{0} \neq 0, \Delta_{0}=0\right)$. Finally, in the charged PC phase the GMP lies at the point $\left(M_{0}=0, \Delta_{0} \neq 0\right)$. [Notice, that in the most general case the coordinates (condensates) $M_{0}$ and $\Delta_{0}$ of the global minimum point depend on chemical potentials.]

In the present subsection we would like to obtain the expression for the quark number (or particle) density $n_{q}$ in the ground state of each phase. Recall that in the most general case this quantity is defined by the relation ${ }^{6}$

$$
n_{q}=-\frac{\partial \Omega^{\mathrm{ren}}\left(M_{0}, \Delta_{0}\right)}{\partial \mu} .
$$

\footnotetext{
${ }^{6}$ The density of baryons $n_{B}$ and the quark number density $n_{q}$ are connected by the relation $n_{q}=3 n_{B}$.
} 
Hence, in the chiral-symmetry-breaking phase we have

$$
\begin{aligned}
\left.n_{q}\right|_{\mathrm{CSB}}= & -\frac{\partial \Omega^{\mathrm{ren}}\left(M_{0} \neq 0, \Delta_{0}=0\right)}{\partial \mu}=-\frac{\partial F_{1}\left(M_{0}\right)}{\partial \mu} \\
= & \frac{\theta\left(\mu+\nu-M_{0}\right)}{\pi} \sqrt{(\mu+\nu)^{2}-M_{0}^{2}} \\
& +\frac{\operatorname{sign}(\mu-\nu) \theta\left(|\mu-\nu|-M_{0}\right)}{\pi} \sqrt{(\mu-\nu)^{2}-M_{0}^{2}},
\end{aligned}
$$

where $\operatorname{sign}(\mathrm{x})$ denotes the sign function and the quantity $F_{1}(M)$ is given in Eq. (36). The quark number density in the charged pion condensation phase can be easily obtained from Eq. (39) by the simple replacement,

$$
\begin{aligned}
\left.n_{q}\right|_{\mathrm{PC}} & =-\frac{\partial \Omega^{\mathrm{ren}}\left(M_{0}=0, \Delta_{0} \neq 0\right)}{\partial \mu}=-\frac{\partial F_{2}\left(\Delta_{0}\right)}{\partial \mu} \\
& =\left.\left\{\left.n_{q}\right|_{\mathrm{CSB}}\right\}\right|_{M_{0} \rightarrow \Delta_{0} ; \nu \leftrightarrow \nu_{5}},
\end{aligned}
$$

which is due to the relation (37). Supposing in Eq. (39) that $M_{0}=0$, one can find the following expression for the particle density in the symmetric phase (of course, we take into account the constraints $\mu \geq 0, \nu \geq 0$ and $\nu_{5} \geq 0$ ):

$$
\left.n_{q}\right|_{\text {SYM }}=\frac{\mu+\nu}{\pi}+\frac{\operatorname{sign}(\mu-\nu)}{\pi}|\mu-\nu|=\frac{2 \mu}{\pi} .
$$

\section{PHASE STRUCTURE AT ZERO TEMPERATURE}

\section{A. The role of the duality symmetry $\mathcal{D}$ [Eq. (18)] of the TDP}

Suppose now that at some fixed particular values of the chemical potentials $\mu, \nu=A$ and $\nu_{5}=B$ the global minimum of the TDP (35) lies at the point, e.g., $\left(M=M_{0} \neq 0\right.$, $\Delta=0$ ). It means that for such fixed values of the chemical potentials the CSB phase is realized in the model. Then it follows from the duality invariance of the TDP (15) [or Eq. (35)] with respect to the transformation $\mathcal{D}$ [Eq. (18)] that the permutation of the chemical potential values (i.e. $\nu=B$ and $\nu_{5}=A$ and the intact value of $\mu$ ) moves the global minimum of the TDP $\Omega^{\text {ren }}(M, \Delta)$ to the point $\left(M=0, \Delta=M_{0}\right)$, which corresponds to the charged PC phase (and vice versa). This is the so-called duality correspondence between CSB and charged PC phases in the framework of the model under consideration. ${ }^{7}$

Hence, the knowledge of a phase of the model (1) at some fixed values of the external free model parameters $\mu, \nu, \nu_{5}$ is sufficient to understand what phase (we call it a

\footnotetext{
${ }^{7}$ It is worth noting that in some $(1+1)$ - and $(2+1)$ dimensional models there is a duality between CSB and superconductivity $[47,48]$.
}

dually conjugated phase) is realized at rearranged values of the external parameters, $\nu \leftrightarrow \nu_{5}$, at fixed $\mu$. Moreover, different physical parameters such as condensates, densities, etc., which characterize both the initial phase and the dually conjugated phase, are connected by the duality transformation $\mathcal{D}$. For example, the chiral condensate of the initial CSB phase at some fixed $\mu, \nu, \nu_{5}$ is equal to the charged-pion condensate of the dually conjugated charged PC phase, in which one should perform the replacement $\nu \leftrightarrow \nu_{5}$. Knowing the particle density $n_{q}$ of the initial CSB phase [see Eq. (39)] as a function of the external chemical potentials $\mu, \nu$ and $\nu_{5}$, one can find the particle density (40) in the dually conjugated charged PC phase by interchanging $\nu$ and $\nu_{5}$ in the initial expression $n_{q}$ for the particle density in the CSB phase (see also Sec. IIIC), etc.

The duality transformation $\mathcal{D}$ of the TDP can also be applied to an arbitrary phase portrait of the model (see below). In particular, it is clear that if we have a most general phase portrait, i.e. the correspondence between any point $\left(\nu, \nu_{5}, \mu\right)$ of the three-dimensional space of external parameters and possible model phases (CSB, charged PC and symmetric phases), then under the duality transformation $\left(\nu \leftrightarrow \nu_{5}, \mathrm{CSB} \leftrightarrow\right.$ charged PC and the symmetrical phase does not change) this phase portrait is mapped to itself, i.e. the most general $\left(\nu, \nu_{5}, \mu\right)$-phase portrait is selfdual. The self-duality of the $\left(\nu, \nu_{5}, \mu\right)$-phase portrait means that the regions of the CSB and charged PC phases in the three-dimensional $\left(\nu, \nu_{5}, \mu\right)$ space are arranged mirror symmetrically with respect to the plane $\nu=\nu_{5}$ of this space. In Sec. IV B, we will present a few sections of this three-dimensional $\left(\nu, \nu_{5}, \mu\right)$-phase portrait of the model by the planes of the form $\mu=$ const, $\nu=$ const and $\nu_{5}=$ const, respectively.

\section{B. Promotion of the dense charged PC phase by $\nu_{\mathbf{5}} \neq 0$}

First of all, we will study the phase structure of the model (1) at different fixed values of the chiral isospin chemical potential $\nu_{5}$. To this end, we determine numerically the global minimum points of the TDPs $F_{1}(M)$ [Eq. (36)] and $F_{2}(\Delta)$ [Eq. (37)] and then compare the minimum values of these functions with the external parameters $\mu, \nu, \nu_{5}$. Moreover, using the expressions (39) and (40), it is possible to find the quark number density $n_{q}$ or baryon density $n_{B}$ (note that $n_{q}=3 n_{B}$ ) inside each phase. As a result, in Fig. 1 we show several $(\nu, \mu)$-phase portraits, corresponding to $\nu_{5}=0, \nu_{5}=0.75 \mathrm{~m}$ and $\nu_{5}=1.3 \mathrm{~m}$, respectively. Recall that $m$ is a free renormalization-invariant mass scale parameter, which appears in the vacuum case of the model after renormalization [see Eqs. (32) and (33)].

The phase portrait of the model in Fig. 1 with $\nu_{5}=0$ was obtained earlier (see e.g. Refs. [16,43]). It is clear from it that at $\nu_{5}=0$ the charged PC phase with nonzero baryon density $n_{B}$ is not realized in the model under consideration. Only the charged PC phase with zero baryon density can be observed at rather small values of $\mu$. (Physically, it means 

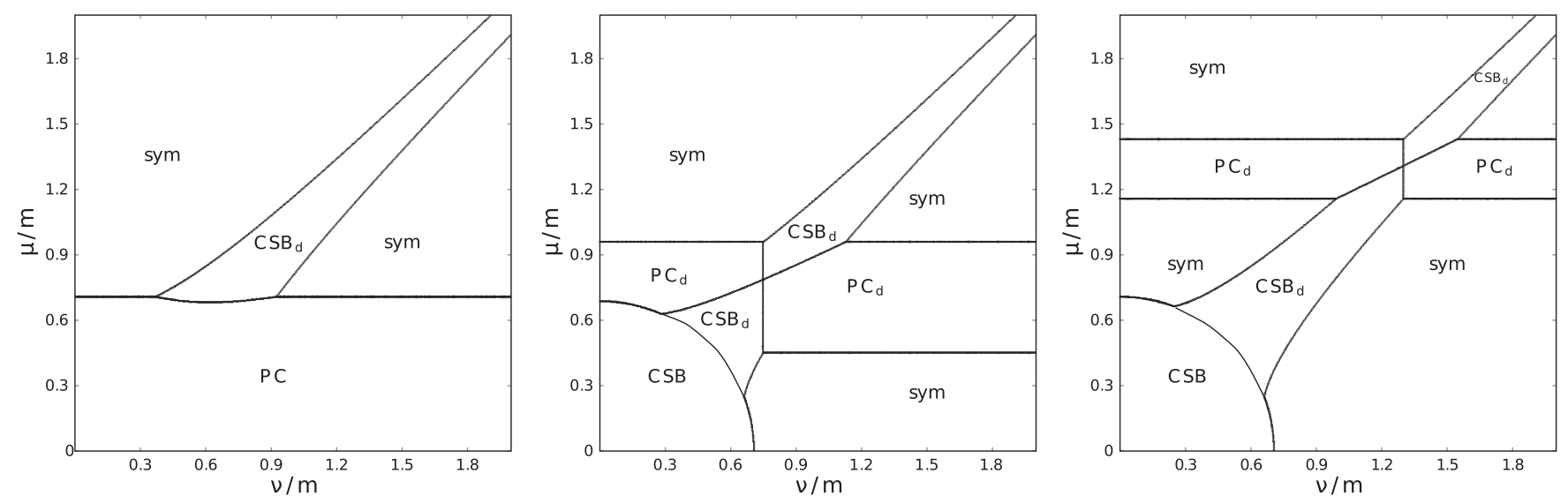

FIG. 1. The $(\nu, \mu)$-phase portraits of the model for different values of the chiral chemical potential $\nu_{5}$ : the case $\nu_{5}=0$ (left panel), the case $\nu_{5}=0.75 \mathrm{~m}$ (middle panel) and the case $\nu_{5}=1.3 \mathrm{~m}$ (right panel). The notations $\mathrm{PC}$ and $\mathrm{PC}_{d}$ mean the charged pion condensation phase with zero and nonzero baryon density, respectively. Analogously, the notations CSB and $\mathrm{CSB}_{d}$ mean the chiral-symmetrybreaking phase with zero and nonzero baryon density, respectively, and SYM denotes the symmetric phase. The parameter $m$ is introduced in Eq. (32). All the lines are solid and denote phase transitions of the first order.

that at $\nu_{5}=0$ the model predicts the charged PC phenomenon in the medium with $n_{B}=0$ only. For example, it might consist of charged pions, etc. But in quark matter with nonzero baryon density the charged PC is forbidden.) Instead, at large values of $\mu$ there exist two phasesthe chiral-symmetry-breaking phase and the symmetrical phase - both with nonzero baryon density, i.e. the model predicts only the CSB or symmetrical phases of dense quark matter at $\nu_{5}=0$. However, as we can see from the other phase diagrams in Fig. 1, at rather high values of $\nu_{5}$, e.g., at $\nu_{5}=0.75 \mathrm{~m}$ or $\nu_{5}=1.3 \mathrm{~m}$, there might appear in the phase portrait a charged PC phase with nonzero baryon density (in Fig. 1 and other figures it is denoted as $\mathrm{PC}_{d}$ ). Hence, in chirally asymmetric (i.e. at $\nu_{5}>0$ ) and dense quark matter the charged PC phenomenon is allowed to exist in the framework of the toy model (1). Thus, we see that $\nu_{5} \neq 0$ is a factor which promotes the charged PC phenomenon in dense quark matter.

Now, suppose that we want to obtain a $\left(\nu_{5}, \mu\right)$-phase portrait of the model at some fixed value $\nu=$ const. In this case there is no need to perform the direct numerical investigations of the TDP (35). In contrast [due to the dual invariance (18) of the model TDP], one can simply make the dual transformation of the $(\nu, \mu)$-phase diagram at the
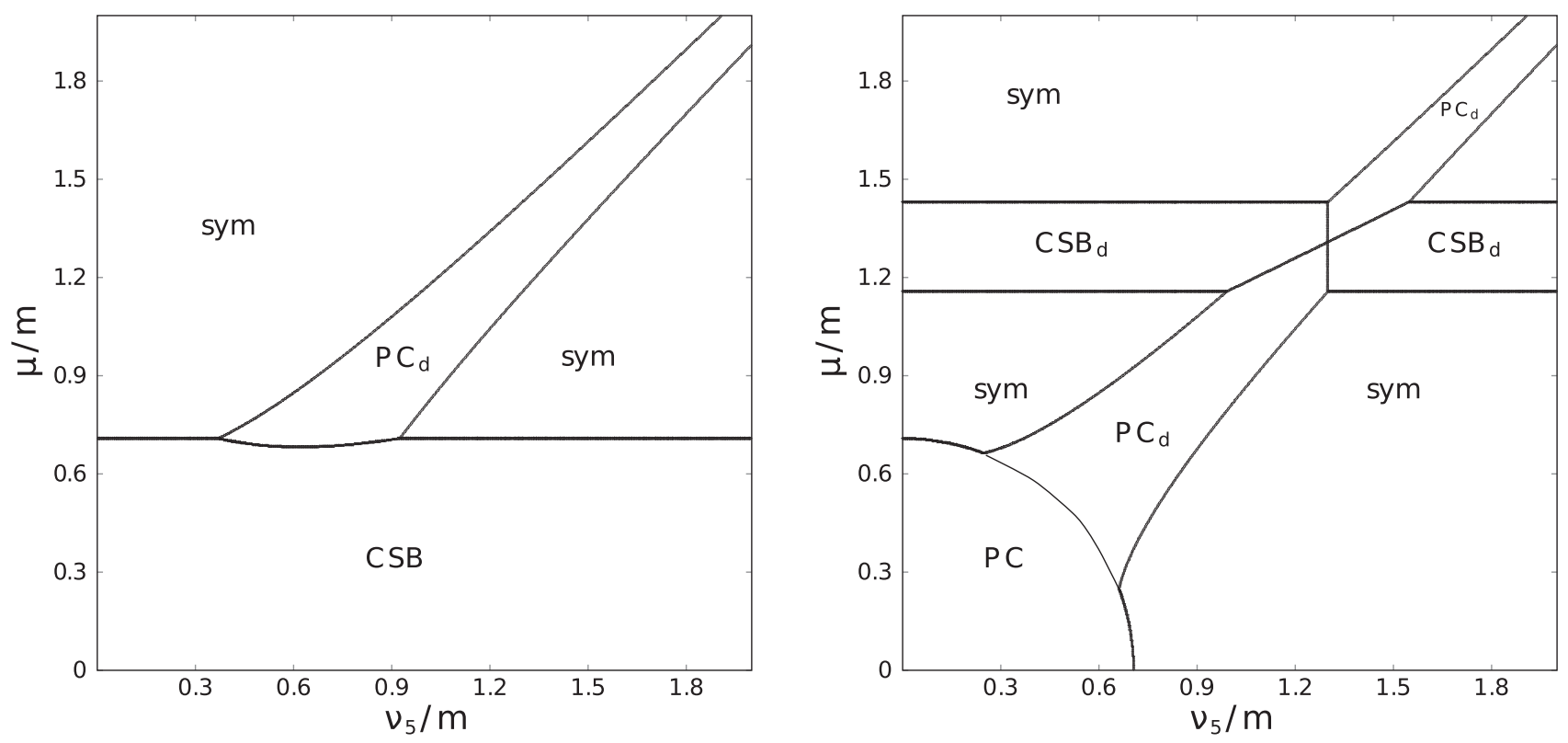

FIG. 2. The $\left(\nu_{5}, \mu\right)$-phase portrait of the model for different values of the isospin chemical potential $\nu$ : the case $\nu=0$ (left panel) and case $\nu=1.3 m$ (right panel). All the lines are the first-order phase transition curves. The notations are the same as in Fig. 1. 

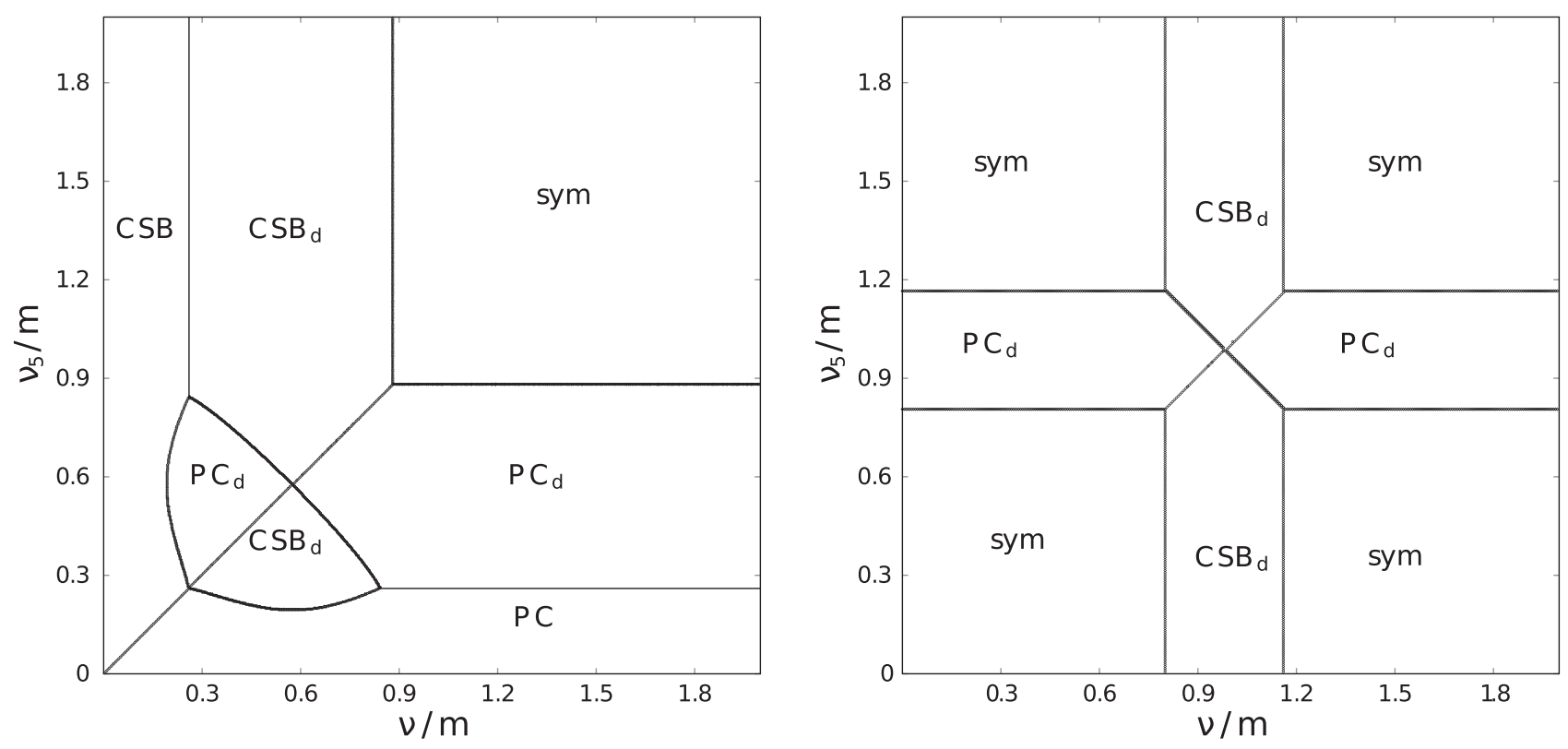

FIG. 3. The $\left(\nu, \nu_{5}\right)$-phase portraits of the model for different values of the quark number chemical potential $\mu$ : the case $\mu=0.65 m$ (left panel) and the case $\mu=m$ (right panel). All the lines are the first-order phase transition curves. The notations are the same as in Fig. 1.

corresponding fixed value $\nu_{5}=$ const. For example, to find the $\left(\nu_{5}, \mu\right)$-phase diagram at $\nu=0$ we should start from the $(\nu, \mu)$ diagram at fixed $\nu_{5}=0$ of Fig. 1 and make the simplest replacement in the notations of this figure: $\nu \rightarrow \nu_{5}$, $\mathrm{PC} \leftrightarrow \mathrm{CSB}, \mathrm{PCd} \leftrightarrow \mathrm{CSBd}$ and the notation "sym" does not change. As a result of this mapping, we obtain the phase diagram of Fig. 2 (left panel) with the $\mathrm{PC}_{d}$ phase. From this phase diagram, we can also draw the interesting conclusion that the $\mathrm{PC}_{d}$ phase can occur in a dense, isotopically symmetric baryonic medium, $\nu=0$, but at the same time $\nu_{5}$ should be nonzero.

In a similar way, to obtain the $\left(\nu_{5}, \mu\right)$-phase diagram, e.g., at $\nu=1.3 m$, it is sufficient to apply the duality transformation to the $(\nu, \mu)$-phase portrait of the model at $\nu_{5}=1.3 \mathrm{~m}$ (see the right panel of Fig. 1). The resulting mapping is the right panel of Fig. 2, etc. It thus supports the above conclusion: the charged PC phenomenon can be realized in chirally asymmetric quark matter with nonzero baryon density.

Finally, let us consider the $\left(\nu, \nu_{5}\right)$-phase diagrams of the model at different fixed values of $\mu$. It is clear from the previous discussions that each of these diagrams is a self-dual one, i.e. the CSB and charged PC phases are arranged symmetrically with respect to the line $\nu=\nu_{5}$ of the $\left(\nu, \nu_{5}\right)$ plane. This fact is confirmed by the $\left(\nu, \nu_{5}\right)$-phase portraits of Fig. 3, obtained by direct numerical analysis of the TDPs $F_{1}(M)$ [Eq. (36)] and $F_{2}(\Delta)$ [Eq. (37)]. Moreover, the phase diagrams of Fig. 3 support once again the main conclusion of our paper: the charged PC phase with nonzero baryon density, i.e. the phase denoted in Figs. 1-3 as $\mathrm{PC}_{d}$, might be realized in the framework of the model (1) only at $\nu_{5} \neq 0$.
We have already noted that the phase structure of this $\mathrm{NJL}_{2}$ model was previously studied in Ref. [50]. And there the conclusion was drawn that the chiral isospin chemical potential $\mu_{15} \equiv 2 \nu_{5}$ generates the charged PC phase in dense quark matter, but only for sufficiently small values of the isospin chemical potential $\mu_{I} \equiv 2 \nu$. However, this result was significantly different from the predictions of the $\mathrm{NJL}_{4}$ model, which were obtained later in Refs. [26,31], where it was stated that the charged PC phase can exist in a chirally asymmetric medium only at sufficiently high values of the isospin chemical potential $\mu_{I}$. Based on this latter result, it is possible to predict that the charged PC phase can be realized in the cores of such astrophysical objects as neutron stars, in which both the isotopic $\mu_{I}$ and chiral isotopic $\mu_{I 5}$ chemical potential can reach large values. (The possibility of the appearance of regions with $\mu_{I 5} \neq 0$ inside neutron stars was discussed in Appendix A of Ref. [26]). In this case, the charged PC phenomenon can have a significant influence on the physical processes in neutron stars and lead to observable effects. However, as noted in the Introduction, the results of Ref. [50] were based on an erroneous expression for the TDP. The correct phase diagrams of the $\mathrm{NJL}_{2}$ model (1) are presented just in the present work, from which it can be seen (see Figs. 1 and 3) that the charged $\mathrm{PC}_{d}$ phase occupies an infinitely long strip along the $\nu$ axis (for some fixed values of $\nu_{5}$ ), which is largely consistent with the predictions of the $\mathrm{NJL}_{4}$ model (see Refs. [26,31]). Thus, we see that two generally different effective models, $\mathrm{NJL}_{2}$ and $\mathrm{NJL}_{4}$, predict qualitatively identical properties of quark matter with chiral isotopic asymmetry, which, in our opinion, enhances the realism of their predictions. 

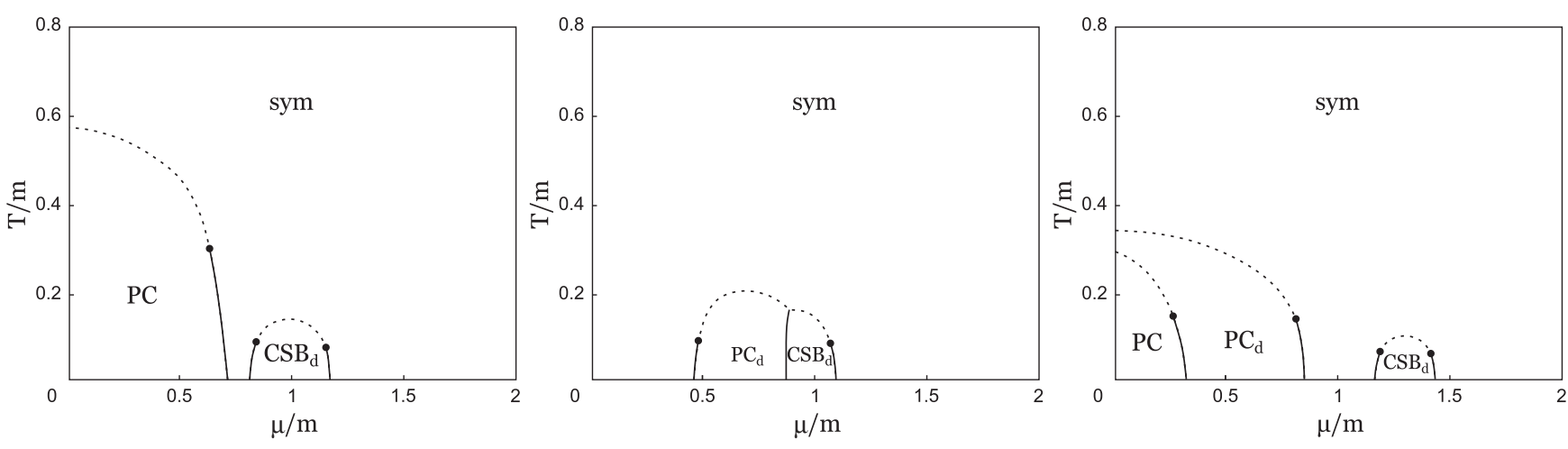

FIG. 4. The $(T, \mu)$-phase portraits of the model for different values of the chemical potentials $\nu$ and $\nu_{5}$ : the case $\nu=m$ and $\nu_{5}=0$ (left panel), the case $\nu=0.9 \mathrm{~m}$ and $\nu_{5}=0.75 \mathrm{~m}$ (middle panel) and the case $\nu=1.3 \mathrm{~m}$ and $\nu_{5}=0.6 \mathrm{~m}$ (right panel). Dashed lines are the second-order phase transition curves, while solid lines are the first-order phase transition curves. All the notations are the same as in Fig. 1. The dots are the tricritical points where phase transitions of two types meet.

\section{PHASE STRUCTURE AT $T \neq 0$}

First of all note that the temperature-dependent TDP $\Omega_{T}(M, \Delta)$ in Eq. (29) is a UV-divergent quantity. While the second term on the right-hand side of this relation is a convergent improper integral that does not contain UV divergences, the first term $\Omega(M, \Delta)$ is a UV-divergent TDP of the model at $T=0$. So both the TDP $\Omega(M, \Delta)$ at $T=0$ and the TDP $\Omega_{T}(M, \Delta)$ at nonzero temperature can be renormalized using the technique presented in the Sec. III B. As a result, one can obtain the following expression for the renormalized TDP of the model at $T \neq 0$ :

$\Omega_{T}^{\mathrm{ren}}(M, \Delta)=\Omega^{\mathrm{ren}}(M, \Delta)-T \sum_{i=1}^{4} \int_{-\infty}^{\infty} \frac{d p_{1}}{2 \pi} \ln \left(1+e^{-\beta\left|p_{0 i}\right|}\right)$,

where $\Omega^{\text {ren }}(M, \Delta)$ is presented in Eq. (35). A numerical algorithm for finding the quasi(anti)particle energies $p_{01}$, $p_{02}, p_{03}$, and $p_{04}$ is elaborated in Appendix A. Based on this, it can be shown numerically that the GMP of the TDP (42) can never be of the form $\left(M_{0} \neq 0, \Delta_{0} \neq 0\right)$. Hence, in order to establish the phase portrait of the model at $T \neq 0$, it is enough to study the projections $F_{1}^{T}(M) \equiv$ $\Omega_{T}^{\text {ren }}(M, \Delta=0)$ and $F_{2}^{T}(\Delta) \equiv \Omega_{T}^{\text {ren }}(M=0, \Delta)$ of the TDP (42) onto the $M$ and $\Delta$ axes, correspondingly. Taking into account the relations (22) and (23) for the quasiparticle energies $p_{0 i}$ at $\Delta=0$ or $M=0$, we have

$$
\begin{aligned}
& F_{1}^{T}(M)=F_{1}(M)-T \sum_{\kappa= \pm} \int_{0}^{\infty} \frac{d q}{\pi} \ln \left(1+e^{-\beta \mid \mu+\nu+\kappa} \sqrt{M^{2}+q^{2}} \mid\right) \\
& -T \sum_{\kappa= \pm} \int_{0}^{\infty} \frac{d q}{\pi} \ln \left(1+e^{-\beta\left|\mu-\nu+\kappa \sqrt{M^{2}+q^{2}}\right|}\right),
\end{aligned}
$$

where $F_{1}(M)$ is given in Eq. (36). Then, the projection $F_{2}^{T}(\Delta)$ of the TDP $\Omega_{T}^{\text {ren }}(M=0, \Delta)$ on the $\Delta$ axis reads

$$
\begin{aligned}
F_{2}^{T}(\Delta)= & \left.F_{1}^{T}(\Delta)\right|_{\nu \leftrightarrow \nu_{5}} \\
= & F_{2}(\Delta)-T \sum_{\kappa= \pm} \int_{0}^{\infty} \frac{d q}{\pi} \ln \left(1+e^{-\beta\left|\mu+\nu_{5}+\kappa \sqrt{\Delta^{2}+q^{2}}\right|}\right) \\
& -T \sum_{\kappa= \pm} \int_{0}^{\infty} \frac{d q}{\pi} \ln \left(1+e^{-\beta\left|\mu-\nu_{5}+\kappa \sqrt{\Delta^{2}+q^{2}}\right|}\right),
\end{aligned}
$$

where $F_{2}(\Delta)$ is given in Eq. (37). By comparing the smallest values of the functions $F_{1}^{T}(M)$ and $F_{2}^{T}(\Delta)$, one can construct the simplest temperature phase diagrams of the model.

First, let us notice that the duality is exact in the chiral limit at any temperature in the framework of the $\mathrm{NJL}_{2}$ model.

The phase diagrams of Fig. 4 are the extensions of some phase portraits at $T=0$ shown in Figs. 1 and 2 to nonzero values of temperature. For example, Fig. 4 (left panel) is the temperature extension of the section $\nu=m$ of the left panel diagram of Fig. 1 and shows at what temperatures the phase structure remains nontrivial. The middle diagram of Fig. 4 is the temperature extension of the section $\nu / m=0.9$ of the middle panel at Fig. 1. Finally, in the right panel of Fig. 4 one can see the temperature extension of the section $\nu_{5} / m=0.6$ of the right panel of Fig. 2. The dashed lines denote second-order phase transitions, but one can note that there are regions of first-order phase transitions at not very large temperatures denoted by the solid lines. The points, where two types of phase transitions meet, are the so-called tricritical points (at the physical point, i.e. at nonzero bare quark mass, they correspond to the critical end points of a phase diagram). Recall that at zero temperature all phase transitions were of first order. So, in Fig. 4 we show three $(\mu, T)$-phase diagrams of the model. Each of them corresponds to a rather high fixed value of the chemical potential $\nu \gtrsim m$, but to substantially not very large fixed values of $\nu_{5}=0,0.6 \mathrm{~m}$ and $0.75 \mathrm{~m}$, respectively. It is clear that the temperature phase diagrams of Fig. 4 also support the fact that the chiral isospin chemical potential $\mu_{I 5}$ promotes the 
creation of a phase with condensation of charged pions in dense quark matter (it is the $\mathrm{PC}_{d}$ phase in Fig. 4). In addition, it is clear from Fig. 4 that the $\mathrm{PC}_{d}$ phase is quite stable with respect to temperature effects and can be realized in dense quark matter up to rather high temperatures. In Fig. 4 (middle and right panels) one can see that the $\mathrm{PC}_{d}$ phase persists up to $T=0.2 m-0.35 m$ respectively, which are comparatively high temperatures. (If one considers $m$ to be the constituent quark mass value in vacuum around $300 \mathrm{MeV}$, then the $\mathrm{PC}_{d}$ phase corresponds to temperatures as high as $100 \mathrm{MeV}$.) This part of the phase diagram is rather interesting because these conditions are realized in various physical scenarios such as in new-born neutron stars (proto-neutron stars) [52], supernovae [53] and neutron star mergers [54] as well as in heavy-ion collisions [55].

Let us recall that, according to the previous study of quark matter in the framework of the $\mathrm{NJL}_{2}$ model (1) (see Ref. [50]) at sufficiently high baryon densities, the $\mathrm{PC}_{d}$ and $\mathrm{CSB}_{d}$ phases could be realized there only in narrow regions of the $\left(\mu_{I}, \mu_{I 5}\right)$-phase diagram, namely (i) at rather large $\mu_{I 5}$ and small $\mu_{I}$ and (ii) at rather large $\mu_{I}$ and rather small $\mu_{I 5}$, respectively. Other regions of the phase diagram were occupied by either the phases without baryon density or a symmetric phase. According to this analysis, in the cores of neutron stars, where there is a large isospin imbalance $\mu_{I}$, only the $\mathrm{CSB}_{d}$ phase can be realized at a not very high chiral imbalance $\mu_{I 5}$, and the $\mathrm{PC}_{d}$ phase was not likely to be present (because it required a rather low isospin imbalance, which is not the case for the neutron stars). In a similar way, the $\mathrm{NJL}_{2}$ analysis forbade the realization of the $\mathrm{PC}_{d}$ phase in heavy-ion collisions where, if one assumes that the chiral imbalance is generated due to the chiral separation effect and is rather large, one needs a large baryon chemical potential $\mu_{B}$ that can be attained only at comparatively low energy and in that regime the isospin imbalance $\mu_{I}$ can not be small enough for the $\mathrm{PC}_{d}$ phase generation. Or if it is more energetic collisions and due to smaller $\mu_{B}$ probably (let us put aside the magnitude of magnetic field) chiral imbalance is small, then isospin imbalance could be not large enough (due to larger energy). (Or conversely for the $\mathrm{CSB}_{d}$ phase, which is probably less interesting but still, if the isospin imbalance is large, then the chiral imbalance should be necessarily small.) Of course, these conditions can easily be attained but still they put some restrictions on the generation of $\mathrm{PC}_{d}$ in heavy-ion collisions. In this paper we show that in the framework of $\mathrm{NJL}_{2}$ at large baryon density (i.e. at large values of $\mu_{B}$ ) the regions occupied by the $\mathrm{PC}_{d}$ and $\mathrm{CSB}_{d}$ phases are rather large (two bands) and they are not confined to the small areas near the $\mu_{I 5}$ and $\mu_{I}$ axes of the $\left(\mu_{I}, \mu_{I 5}\right)$-phase diagram. This leads to the possible generation of charged pion condensates in dense quark matter with large isospin imbalance, for example in the cores of neutron stars. The possibility of generating the $\mathrm{PC}_{d}$ phase in heavy-ion collisions is less bounded by various constraints and the values of different imbalances. So one can see that the possibility of charged pion condensation in dense quark matter was underestimated in Ref. [50] and is actually a more considerable result of the $\mathrm{NJL}_{2}$ model predictions.

Finally, recall that in the model (1) there is a duality between the CSB and charged PC phenomena. In this case it is possible, relying on the phase portraits of Fig. 4, to get an idea of the $(\mu, T)$-phase structure of the model, when $\nu$ and $\nu_{5}$ are fixed in a qualitatively different way than in Fig. 4 area of the chemical potentials. Namely, when $\nu_{5}$ is fixed at rather high, but $\nu$ is fixed at not very high values, respectively. For example, if we apply the duality transformation in which $\nu \leftrightarrow \nu_{5}, \mathrm{CSB} \leftrightarrow \mathrm{PC}$ and CSBd $\leftrightarrow \mathrm{PCd}$ to Fig. 4 (left panel), we can obtain the $(\mu, T)$-phase portrait of the model at $\nu_{5}=m$ and $\nu=0$. In this new $(\mu, T)$-phase diagram, one can find in the vicinity of the point $\mu=m$ a charged PC phase with nonzero baryon density, which in itself is already an interesting fact since in this case there is no isotopic asymmetry $(\nu=0)$. In a similar way, one can construct the duality mapping of other phase portraits in Fig. 4.

\section{SUMMARY AND CONCLUSIONS}

In this paper the phase structure of the massless $\mathrm{NJL}_{2}$ model (1) with two quark flavors was investigated in the large- $N_{c}$ limit in the presence of baryon $\mu_{B}$, isospin $\mu_{I}$ and chiral isospin $\mu_{I 5}$ chemical potentials. The particular case with $\mu_{I 5}=0$ was solved earlier in Refs. $[16,42,43]$, where it was shown that the toy model (1) does not predict a charged PC phase of dense and isotopically asymmetric quark matter. The case $\mu_{I 5} \neq 0$ was first investigated in Ref. [50], where the phase structure of this $\mathrm{NJL}_{2}$ model was studied at $T=0$. It was shown there that the charged PC phase with nonzero baryon density (this phase is denoted by the symbol $\mathrm{PC}_{d}$ ), prohibited at $\mu_{I 5}=0$, appears at rather large values of $\mu_{15} \neq 0$, and hence, it was concluded that chiral isospin asymmetry (i.e. $\mu_{I 5} \neq 0$ ) can generate a charged PC phenomenon in dense quark matter. (Note that two other known factors promoting a charged PC phase in dense quark matter are a finite volume [16] and the possible spatial inhomogeneity of condensates [17].)

After this prediction was made in the framework of the $\mathrm{NJL}_{2}$ model (1), the phase structure of a more realistic $(3+1)$-dimensional NJL model with nonzero $\mu_{B}, \mu_{I}$ and $\mu_{I 5}$ was investigated in Refs. [26,31] and similar results were obtained, i.e., it was shown in these papers that the chiral isotopic chemical potential $\mu_{I 5}$ (as well as the chiral chemical potential $\mu_{5}$ [31]) generates charged PC phenomenon in dense quark matter. The results were qualitatively similar, but one feature was quite different, namely it was shown that the $\mathrm{PC}_{d}$ phase can be generated only at rather small isospin chemical potential $\mu_{I}$ values in the $\mathrm{NJL}_{2}$ model and for a wide range of $\mu_{I}$ values in the $\mathrm{NJL}_{4}$ model. 
In the present paper we noted that in Ref. [50] a miscalculation was performed in the calculation of the expression for the TDP of the $\mathrm{NJL}_{2}$ model, and here the corrected and enlarged consideration was presented. In addition, we included in the consideration the case of nonzero temperature. It is shown that although the right expression for the TDP is very different from the original one of Ref. [50] (the right one is much simpler), qualitatively the basic features and the main predictions of the $\mathrm{NJL}_{2}$ model analysis turned out to be similar.

(i) Namely, the main conclusion that chiral isospin imbalance generates charged pion condensation in dense and cold $(T=0)$ quark matter remains valid. Moreover, it is quite interesting that the $\mathrm{PC}_{d}$ phase can occur in a dense, isotopically symmetric baryonic medium, $\nu=0$, but at the same time $\nu_{5}$ should be nonzero (see the left panel of Fig. 2).

(ii) This generation of the $\mathrm{PC}_{d}$ phase by $\nu_{5}$ not only remains valid, but is also greatly enhanced and the $\mathrm{PC}_{d}$ phase occupies a long strip along the $\mu_{I}$ axis of different phase portraits (see Figs. 1-3) of the $\mathrm{NJL}_{2}$ model, instead of a small and compact part of the phase diagram at not too large values of the isospin chemical potential as in Ref. [50].

(iii) The possibility for the generation of charged pion condensation in dense quark matter was underestimated in Ref. [50] and is actually more considerable. It can be realized in real physical situations such as heavy-ion collisions or compact stars (see the discussion in Sec. V).

(iv) Moreover, it was also shown in the present paper that in the leading order of the large- $N_{c}$ expansion there is a duality correspondence between CSB and charged PC phenomena. The simplest and most obvious manifestation of this phenomenon is that in each $\left(\mu_{I}, \mu_{I 5}\right)$-phase diagram of the model (see, e.g., Fig. 3), each CSB phase is located mirror-symmetrically to some charged PC phase with respect to the line $\mu_{I}=\mu_{I 5}$ (and vice versa).

(v) It has been shown that in the chiral limit duality stays the exact symmetry of the phase diagram of dense quark matter even at very high temperatures as long as the lowenergy effective NJL model description is valid, and can be used for phase structure investigations.

(vi) It has also been demonstrated that the predicted generation of charged pion condensation by a chiral isospin imbalance in dense and cold $(T=0)$ quark matter remains valid even at temperatures as high as several dozens or even a hundred $\mathrm{MeV}$. These conditions are rather pertinent in proto-neutron stars [52], supernovae [53] and neutron star mergers [54] as well as in heavy-ion collisions [55].

So this consideration shows that the possibility of charged pion condensation in dense quark matter at zero temperature was previously underestimated and it is more likely to happen in compact stars. Additionally, it was shown that this phenomenon can take place even at rather high temperatures, which makes it feasible in various physical situations such as heavy-ion collisions, new-born neutron stars (proto-neutron stars), supernovae as well as neutron star mergers.

Taking into account earlier investigations [31], we see that within the framework of the two different effective models - the $\mathrm{NJL}_{4}$ and $\mathrm{NJL}_{2}$ models - quite similar properties (i.e., similar phase portraits) of dense and chirally asymmetric quark matter are predicted. This fact increases our confidence that in the cores of neutron stars, where $\mu_{B}$ and $\mu_{I}$ can reach significant values, the effect of charged PC can be realized. Then, to explain the similarity between the phase diagrams of these two effective models, two circumstances can be taken into account. (i) Under the influence of a strong magnetic field in the depths of neutron stars, regions with a nonzero chiral isotopic density (with $\mu_{I 5} \neq 0$ ) can appear (for more details, see Appendix A of Refs. [26,27]). (ii) Just in the presence of a strong magnetic field, the dynamics of fermion pairing is essentially $(1+1)$ dimensional [46]. Therefore, the phase portraits of dense quark matter at $\mu_{I 5} \neq 0$ obtained in framework of the $\mathrm{NJL}_{4}$ and $\mathrm{NJL}_{2}$ models are qualitatively the same. However, it is worth noting that $(1+1)$ dimensional models are not comprehensive. Thus, phase transformations in real dense media with a strong magnetic field are still available for description in a two-dimensional framework, which cannot be said of many other physical properties. For example, the influence of the charged PC phase on various processes in neutron stars, such as transport processes etc., cannot be considered within the $\mathrm{NJL}_{2}$ model. It is not the subject of the present paper, and will be investigated in the framework of a more realistic model elsewhere.

\section{ACKNOWLEDGMENTS}

R. N. Z. is grateful for support of the Foundation for the Advancement of Theoretical Physics and Mathematics BASIS grant and the Russian Science Foundation under the Contract No. 19-72-00077.

\section{APPENDIX A: EVALUATION OF THE ROOTS OF THE POLYNOMIAL $\boldsymbol{P}_{4}\left(p_{0}\right)(16)$}

It is very convenient to present the fourth-order polynomial (16) of the variable $\eta \equiv p_{0}+\mu$ as a product of two second-order polynomials (this method was proposed in Ref. [56]), i.e. we assume that

$$
\begin{aligned}
& \eta^{4}-2 a \eta^{2}-b \eta+c=\left(\eta^{2}+r \eta+q\right)\left(\eta^{2}-r \eta+s\right) \\
& =\left[\left(\eta+\frac{r}{2}\right)^{2}+q-\frac{r^{2}}{4}\right]\left[\left(\eta-\frac{r}{2}\right)^{2}+s-\frac{r^{2}}{4}\right] \\
& \equiv\left(\eta-\eta_{1}\right)\left(\eta-\eta_{2}\right)\left(\eta-\eta_{3}\right)\left(\eta-\eta_{4}\right),
\end{aligned}
$$

where $r, q$ and $s$ are some real-valued quantities, such that [see the relations (17)] 


$$
\begin{aligned}
-2 a & \equiv-2\left(M^{2}+\Delta^{2}+p_{1}^{2}+\nu^{2}+\nu_{5}^{2}\right)=s+q-r^{2}, \\
-b & \equiv-8 p_{1} \nu \nu_{5}=r s-q r, \\
c & \equiv a^{2}-4 p_{1}^{2}\left(\nu^{2}+\nu_{5}^{2}\right)-4 M^{2} \nu^{2}-4 \Delta^{2} \nu_{5}^{2}-4 \nu^{2} \nu_{5}^{2}=s q .
\end{aligned}
$$

In the most general case, i.e. at $M \geq 0, \Delta \geq 0, \nu \geq 0, \nu_{5} \geq 0$ and for arbitrary values of $p_{1}$, one can solve the system of equations (A3) with respect to $q, s, r$ and find

$$
\begin{aligned}
q & =\frac{1}{2}\left(-2 a+R+\frac{b}{\sqrt{R}}\right), \quad s=\frac{1}{2}\left(-2 a+R-\frac{b}{\sqrt{R}}\right), \\
r & =\sqrt{R},
\end{aligned}
$$

where $R$ is an arbitrary real positive solution of the equation

$$
X^{3}+A X=B X^{2}+C
$$

with respect to a variable $X$, and

$$
\begin{aligned}
& A=4 a^{2}-4 c=16\left[\nu_{5}^{2} \Delta^{2}+M^{2} \nu^{2}+\nu_{5}^{2} \nu^{2}+p_{1}^{2}\left(\nu^{2}+\nu_{5}^{2}\right)\right], \\
& B=4 a=4\left(M^{2}+\Delta^{2}+\nu^{2}+\nu_{5}^{2}+p_{1}^{2}\right), \\
& C=b^{2}=\left(8 \nu_{5} \nu p_{1}\right)^{2} .
\end{aligned}
$$

Finding (numerically) the quantities $q, s$ and $r$, it is possible to obtain from Eq. (A2) the roots $\eta_{i}$ :

$$
\begin{array}{ll}
\eta_{1}=-\frac{r}{2}+\sqrt{\frac{r^{2}}{4}-q}, & \eta_{2}=\frac{r}{2}+\sqrt{\frac{r^{2}}{4}-s}, \\
\eta_{3}=-\frac{r}{2}-\sqrt{\frac{r^{2}}{4}-q}, & \eta_{4}=\frac{r}{2}-\sqrt{\frac{r^{2}}{4}-s .}
\end{array}
$$

A numerical investigation shows that in the most general case the discriminant of the third-order algebraic equation (A5), i.e. the quantity $18 A B C-4 B^{3} C+A^{2} B^{2}-$ $4 A^{3}-27 C^{2}$, is always non-negative. So Eq. (A5) vs $X$ has three real solutions $R_{1}, R_{2}$ and $R_{3}$ (this fact was presented in Ref. [56]). Moreover, since the coefficients $A, B$ and $C$ [Eq. (A6)] are non-negative, it is clear that, due to the form of Eq. (A5), all its roots $R_{1}, R_{2}$ and $R_{3}$ are also non-negative quantities (usually, they are positive and different). So we are free to choose the quantity $R$ from Eq. (A4) as one of the positive solutions $R_{1}, R_{2}$ or $R_{3}$. In each case, i.e. for $R=R_{1}, R=R_{2}$, or $R=R_{3}$, we will obtain the same set of roots (A7) (possibly rearranged), which depends only on $\nu$, $\nu_{5}, M, \Delta$ and $p_{1}$, and does not depend on the choice of $R$. Due to the relations (A2)-(A7), one can find numerically (at fixed values of $\mu, \nu, \nu_{5}, M, \Delta$ and $p_{1}$ ) the roots $\eta_{i}=$ $p_{0 i}+\mu$ [Eq. (A7)] and, as a result, numerically investigate the TDP (25).

Analyzing the relations (A1)-(A7), we can draw another important conclusion concerning the properties of the roots $\eta_{i}$ of the polynomial (A1) with respect to the duality transformations $\mathcal{D}$ [Eq. (18)]. It is clear that the coefficients of this polynomial are invariant under the action of $\mathcal{D}$, so each root $\eta_{i}$ [Eq. (A7)] is transformed under the dual mapping of $\mathcal{D}$ to itself, if the quantity $R$ from Eq. (A4) remains intact under the action of $\mathcal{D}$ [see Eq. (A11)]. Note that in our work the quantity $R$ is chosen with exactly this property. However, in the most general case (when $R$ is selected to not be invariant under the $\mathcal{D}$ transformation) the set of roots $\eta_{i}$ also remains unchanged (with the cost of a possible rearrangement of these roots) under the action of $\mathcal{D}$. Hence, the TDPs of Eqs. (29), (35) etc. are invariant with respect to the duality transformation (18).

On the basis of the relations (A1)-(A7) let us consider the asymptotic behavior of the quasiparticle energies $p_{0 i}$ at $p_{1} \rightarrow \pm \infty$. First of all, we start from the asymptotic analysis of the roots $R_{1,2,3}$ of Eq. (A5) at $p_{1} \rightarrow \pm \infty$,

$$
\begin{gathered}
R_{1}=4 \nu^{2}-\frac{4 \Delta^{2} \nu^{2}}{p_{1}^{2}}+\mathcal{O}\left(1 / p_{1}^{4}\right), \\
R_{2}=4 \nu_{5}^{2}-\frac{4 M^{2} \nu_{5}^{2}}{p_{1}^{2}}+\mathcal{O}\left(1 / p_{1}^{4}\right), \\
R_{3}=4 p_{1}^{2}+4\left(M^{2}+\Delta^{2}\right)+\frac{4\left(\nu_{5}^{2} M^{2}+\nu^{2} \Delta^{2}\right)}{p_{1}^{2}}+\mathcal{O}\left(1 / p_{1}^{4}\right) .
\end{gathered}
$$

It is clear from these relations that $R_{3}$ is invariant under the duality transformation (18), whereas $R_{1} \leftrightarrow R_{2}$. Then, using for example $R_{3}$ [Eq. (A10)] as the quantity $R$ in Eqs. (A4) and (A7), one can get the asymptotics of the quasiparticle energies $p_{0 i} \equiv \eta_{i}-\mu$ at $p_{1} \rightarrow \pm \infty$,

$$
\begin{aligned}
& p_{01}=-\left|p_{1}\right|-\mu+\left|\nu_{5}-\nu\right|-\frac{\Delta^{2}+M^{2}}{2\left|p_{1}\right|}+\mathcal{O}\left(1 / p_{1}^{2}\right), \\
& p_{02}=\left|p_{1}\right|-\mu+\nu_{5}+\nu+\frac{\Delta^{2}+M^{2}}{2\left|p_{1}\right|}+\mathcal{O}\left(1 / p_{1}^{2}\right), \\
& p_{03}=-\left|p_{1}\right|-\mu-\left|\nu_{5}-\nu\right|-\frac{\Delta^{2}+M^{2}}{2\left|p_{1}\right|}+\mathcal{O}\left(1 / p_{1}^{2}\right), \\
& p_{04}=\left|p_{1}\right|-\mu-\nu_{5}-\nu+\frac{\Delta^{2}+M^{2}}{2\left|p_{1}\right|}+\mathcal{O}\left(1 / p_{1}^{2}\right) .
\end{aligned}
$$

Finally, it follows from Eq. (A11) that at $p_{1} \rightarrow \pm \infty$

$$
\begin{aligned}
& \left|p_{01}\right|+\left|p_{02}\right|+\left|p_{03}\right|+\left|p_{04}\right| \\
& \quad=4\left|p_{1}\right|+\frac{2\left(\Delta^{2}+M^{2}\right)}{\left|p_{1}\right|}+\mathcal{O}\left(1 / p_{1}^{2}\right) .
\end{aligned}
$$

For the purposes of the renormalization of the TDP (25), it is very important that the leading terms of this asymptotic behavior do not depend on different chemical potentials, i.e. the quantity $\sum_{i=1}^{4}\left|p_{0 i}\right|$ at $\mu=\nu=\nu_{5}=0$ has the same 
asymptotic expansion (A12). Furthermore, there is an exact expression for this sum,

$$
\begin{aligned}
& \left.\left(\left|p_{01}\right|+\left|p_{02}\right|+\left|p_{03}\right|+\left|p_{04}\right|\right)\right|_{\mu=\nu=\nu_{5}=0} \\
& \quad=4 \sqrt{M^{2}+\Delta^{2}+p_{1}^{2}} .
\end{aligned}
$$

We would like to emphasize once again that the asymptotic behavior (A12) does not depend on which of the roots $R_{1}$, $R_{2}$ or $R_{3}$ of Eq. (A5) is taken as the quantity $R$ in the relations (A4). Finally, it is important to note that the quantity $\sum_{i=1}^{4}\left|p_{0 i}\right|$ is not an even function with respect to the momentum $p_{1}$. [This statement is supported, in particular, by the relation (B1) of Appendix B.] Indeed, the polynomial (A1) is not invariant under the transformation $p_{1} \rightarrow-p_{1}$ (since its coefficient $b$ changes). So each root $\eta_{i}$ and the sum $\sum_{i=1}^{4}\left|p_{0 i}\right|$ are changed under this transformation. (In contrast, if $\nu=0$ or $\nu_{5}=0$ then this sum is an even function of $p_{1}$.) Unfortunately, the opposite, incorrect assertion was made in Ref. [50], which led to an incorrect expression for the TDPs $F_{1}(M)$ and $F_{2}(\Delta)$.

\section{APPENDIX B: DERIVATION OF THE RELATION (36)}

If $\Delta=0$ and $M \neq 0$, then the quasiparticle energies $p_{0 i}$ are presented in Eq. (22). So

$$
\begin{aligned}
& \left.\left(\left|p_{01}\right|+\left|p_{02}\right|+\left|p_{03}\right|+\left|p_{04}\right|\right)\right|_{\Delta=0} \\
& =\sum_{\kappa= \pm}\left(\left|-\mu+\kappa \nu+\sqrt{M^{2}+\left(p_{1}+\kappa \nu_{5}\right)^{2}}\right|+\left|-\mu+\kappa \nu-\sqrt{M^{2}+\left(p_{1}+\kappa \nu_{5}\right)^{2}}\right|\right) \\
& =2 \sum_{\kappa= \pm}\left\{\sqrt{M^{2}+\left(p_{1}+\kappa \nu_{5}\right)^{2}}+\left(\mu-\kappa \nu-\sqrt{M^{2}+\left(p_{1}+\kappa \nu_{5}\right)^{2}}\right) \theta\left(\mu-\kappa \nu-\sqrt{M^{2}+\left(p_{1}+\kappa \nu_{5}\right)^{2}}\right)\right. \\
& \left.\quad+\left(\kappa \nu-\mu-\sqrt{M^{2}+\left(p_{1}+\kappa \nu_{5}\right)^{2}}\right) \theta\left(\kappa \nu-\mu-\sqrt{M^{2}+\left(p_{1}+\kappa \nu_{5}\right)^{2}}\right)\right\},
\end{aligned}
$$

where we have taken into account the well-known relations $|x|=x \theta(x)-x \theta(-x)$ and $\theta(x)=1-\theta(-x)$. Hence, the expression (35) at $\Delta=0$ and $M \neq 0$ can be presented in the following form:

$$
F_{1}(M) \equiv \Omega^{\mathrm{ren}}(M, \Delta=0)=-\frac{M^{2}}{2 \pi}+\frac{M^{2}}{2 \pi} \ln \left(\frac{M^{2}}{m^{2}}\right)-U-V,
$$

where

$$
\begin{aligned}
& U= \int_{-\infty}^{\infty} \frac{d p_{1}}{2 \pi}\left\{\sqrt{M^{2}+\left(p_{1}+\nu_{5}\right)^{2}}+\sqrt{M^{2}+\left(p_{1}-\nu_{5}\right)^{2}}-2 \sqrt{M^{2}+p_{1}^{2}}\right\}=\frac{\nu_{5}^{2}}{\pi}, \\
& V=\sum_{\kappa= \pm} \int_{-\infty}^{\infty} \frac{d p_{1}}{2 \pi}\left\{\left(\mu-\kappa \nu-\sqrt{M^{2}+\left(p_{1}+\kappa \nu_{5}\right)^{2}}\right) \theta\left(\mu-\kappa \nu-\sqrt{M^{2}+\left(p_{1}+\kappa \nu_{5}\right)^{2}}\right)\right. \\
&\left.+\left(\kappa \nu-\mu-\sqrt{M^{2}+\left(p_{1}+\kappa \nu_{5}\right)^{2}}\right) \theta\left(\kappa \nu-\mu-\sqrt{M^{2}+\left(p_{1}+\kappa \nu_{5}\right)^{2}}\right)\right\} \\
&=\int_{-\infty}^{\infty} \frac{d p_{1}}{2 \pi}\left(\mu-\nu-\sqrt{M^{2}+\left(p_{1}+\nu_{5}\right)^{2}}\right) \theta\left(\mu-\nu-\sqrt{M^{2}+\left(p_{1}+\nu_{5}\right)^{2}}\right) \\
&+\int_{-\infty}^{\infty} \frac{d p_{1}}{2 \pi}\left(\nu-\mu-\sqrt{M^{2}+\left(p_{1}+\nu_{5}\right)^{2}}\right) \theta\left(\nu-\mu-\sqrt{M^{2}+\left(p_{1}+\nu_{5}\right)^{2}}\right) \\
&+\int_{-\infty}^{\infty} \frac{d p_{1}}{2 \pi}\left(\mu+\nu-\sqrt{M^{2}+\left(p_{1}-\nu_{5}\right)^{2}}\right) \theta\left(\mu+\nu-\sqrt{M^{2}+\left(p_{1}-\nu_{5}\right)^{2}}\right) .
\end{aligned}
$$

Notice that a calculation of the convergent improper integral $U$ [Eq. (B3)] can be found, e.g., in Appendix C of Ref. [16]. Moreover, when summing in Eq. (B4) over $\kappa= \pm$, we have taken into account that $\mu \geq 0$ and $\nu \geq 0$. So there are only three integrals in the expression (B5). Due to the presence of the step function $\theta(x)$, each integral in Eq. (B5) is indeed a proper one. Let us denote the sum of the first two integrals of Eq. (B5) as $V_{1}$ and the last integral as $V_{2}$, i.e. $V=V_{1}+V_{2}$. Then, it is evident that 


$$
\begin{gathered}
V_{1}=\int_{-\infty}^{\infty} \frac{d p_{1}}{2 \pi}\left(|\mu-\nu|-\sqrt{M^{2}+\left(p_{1}+\nu_{5}\right)^{2}}\right) \theta\left(|\mu-\nu|-\sqrt{M^{2}+\left(p_{1}+\nu_{5}\right)^{2}}\right), \\
V_{2}=\int_{-\infty}^{\infty} \frac{d p_{1}}{2 \pi}\left(\mu+\nu-\sqrt{M^{2}+\left(p_{1}-\nu_{5}\right)^{2}}\right) \theta\left(\mu+\nu-\sqrt{M^{2}+\left(p_{1}-\nu_{5}\right)^{2}}\right) .
\end{gathered}
$$

In these relations the region of integration over $p_{1} \in(-\infty,+\infty)$ can be transformed to the region $p_{1} \in(0,+\infty)$.

$$
\begin{gathered}
V_{1}=\int_{0}^{\infty} \frac{d p_{1}}{2 \pi}\left(|\nu-\mu|-\sqrt{M^{2}+\left(p_{1}+\nu_{5}\right)^{2}}\right) \theta\left(|\nu-\mu|-\sqrt{M^{2}+\left(p_{1}+\nu_{5}\right)^{2}}\right) \\
+\int_{0}^{\infty} \frac{d p_{1}}{2 \pi}\left(|\nu-\mu|-\sqrt{M^{2}+\left(p_{1}-\nu_{5}\right)^{2}}\right) \theta\left(|\nu-\mu|-\sqrt{M^{2}+\left(p_{1}-\nu_{5}\right)^{2}}\right), \\
V_{2}=\int_{0}^{\infty} \frac{d p_{1}}{2 \pi}\left(\mu+\nu-\sqrt{M^{2}+\left(p_{1}-\nu_{5}\right)^{2}}\right) \theta\left(\mu+\nu-\sqrt{M^{2}+\left(p_{1}-\nu_{5}\right)^{2}}\right) \\
+\int_{0}^{\infty} \frac{d p_{1}}{2 \pi}\left(\mu+\nu-\sqrt{M^{2}+\left(p_{1}+\nu_{5}\right)^{2}}\right) \theta\left(\mu+\nu-\sqrt{M^{2}+\left(p_{1}+\nu_{5}\right)^{2}}\right) \equiv V_{2-}+V_{2+},
\end{gathered}
$$

where $V_{2-}$ and $V_{2+}$ denote the first and the second integral of Eq. (B9), respectively. Carrying out in the integrals of Eq. (B9) the change of variables, $q=p_{1}+\nu_{5}$ for $V_{2+}$ and $q=p_{1}-\nu_{5}$ for $V_{2-}$, respectively, we have

$$
\begin{aligned}
V_{2+} & =\int_{\nu_{5}}^{\infty} \frac{d q}{2 \pi}\left(\mu+\nu-\sqrt{M^{2}+q^{2}}\right) \theta\left(\mu+\nu-\sqrt{M^{2}+q^{2}}\right) \\
& =\left(\int_{0}^{\infty}-\int_{0}^{\nu_{5}}\right) \frac{d q}{2 \pi}\left(\mu+\nu-\sqrt{M^{2}+q^{2}}\right) \theta\left(\mu+\nu-\sqrt{M^{2}+q^{2}}\right) \\
V_{2-} & =\int_{-\nu_{5}}^{\infty} \frac{d q}{2 \pi}\left(\mu+\nu-\sqrt{M^{2}+q^{2}}\right) \theta\left(\mu+\nu-\sqrt{M^{2}+q^{2}}\right) \\
& =\left(\int_{0}^{\infty}+\int_{0}^{\nu_{5}}\right) \frac{d q}{2 \pi}\left(\mu+\nu-\sqrt{M^{2}+q^{2}}\right) \theta\left(\mu+\nu-\sqrt{M^{2}+q^{2}}\right) .
\end{aligned}
$$

Hence,

$$
\begin{aligned}
V_{2} & \equiv V_{2+}+V_{2-}=\int_{0}^{\infty} \frac{d q}{\pi}\left(\mu+\nu-\sqrt{M^{2}+q^{2}}\right) \theta\left(\mu+\nu-\sqrt{M^{2}+q^{2}}\right) \\
& =\frac{\theta(\mu+\nu-M)}{2 \pi}\left((\mu+\nu) \sqrt{(\mu+\nu)^{2}-M^{2}}-M^{2} \ln \frac{\mu+\nu+\sqrt{(\mu+\nu)^{2}-M^{2}}}{M}\right) .
\end{aligned}
$$

In a similar way it is possible to show that

$$
\begin{aligned}
V_{1} & =\int_{0}^{\infty} \frac{d q}{\pi}\left(|\mu-\nu|-\sqrt{M^{2}+q^{2}}\right) \theta\left(|\mu-\nu|-\sqrt{M^{2}+q^{2}}\right) \\
& =\frac{\theta(|\mu-\nu|-M)}{2 \pi}\left(|\mu-\nu| \sqrt{(\mu-\nu)^{2}-M^{2}}-M^{2} \ln \frac{|\mu-\nu|+\sqrt{(\mu-\nu)^{2}-M^{2}}}{M}\right) .
\end{aligned}
$$

Since $V=V_{1}+V_{2}$ and $U$ is given in Eq. (B3), we have for the TDP $F_{1}(M)$ [Eq. (B2)] the expression (36). 
[1] Y. Nambu and G. Jona-Lasinio, Phys. Rev. 122, 345 (1961).

[2] M. Asakawa and K. Yazaki, Nucl. Phys. A504, 668 (1989); D. Ebert, H. Reinhardt, and M. K. Volkov, Prog. Part. Nucl. Phys. 33, 1 (1994).

[3] D. Ebert, K. G. Klimenko, M. A. Vdovichenko, and A. S. Vshivtsev, Phys. Rev. D 61, 025005 (1999); D. Ebert and K. G. Klimenko, Nucl. Phys. A728, 203 (2003).

[4] D. P. Menezes, M. B. Pinto, S. S. Avancini, A. P. Martinez, and C. Providencia, Phys. Rev. C 79, 035807 (2009); A. Ayala, A. Bashir, A. Raya, and A. Sanchez, Phys. Rev. D 80, 036005 (2009); S. Fayazbakhsh and N. Sadooghi, Phys. Rev. D 90, 105030 (2014); E. J. Ferrer, V. de la Incera, J. P. Keith, I. Portillo, and P. P. Springsteen, Phys. Rev. C 82, 065802 (2010); H. Kohyama, D. Kimura, and T. Inagaki, Nucl. Phys. B896, 682 (2015).

[5] A. J. Mizher, M. N. Chernodub, and E. S. Fraga, Phys. Rev. D 82, 105016 (2010); B. Chatterjee, H. Mishra, and A. Mishra, Phys. Rev. D 84, 014016 (2011).

[6] F. Preis, A. Rebhan, and A. Schmitt, J. High Energy Phys. 03 (2011) 033; M. D’Elia and F. Negro, Phys. Rev. D 83, 114028 (2011); E. V. Gorbar, V. A. Miransky, and I. A. Shovkovy, arXiv:1111.3401.

[7] M. Buballa, Phys. Rep. 407, 205 (2005); I. A. Shovkovy, Found. Phys. 35, 1309 (2005); M. G. Alford, A. Schmitt, K. Rajagopal, and T. Schäfer, Rev. Mod. Phys. 80, 1455 (2008).

[8] D. Blaschke, D. Ebert, K. G. Klimenko, M. K. Volkov, and V. L. Yudichev, Phys. Rev. D 70, 014006 (2004); T. Fujihara, D. Kimura, T. Inagaki, and A. Kvinikhidze, Phys. Rev. D 79, 096008 (2009).

[9] E. J. Ferrer and V. de la Incera, Phys. Rev. D 76, 045011 (2007); S. Fayazbakhsh and N. Sadooghi, Phys. Rev. D 82, 045010 (2010); 83, 025026 (2011).

[10] D. T. Son and M. A. Stephanov, Phys. At. Nucl. 64, 834 (2001); M. Loewe and C. Villavicencio, Phys. Rev. D 67, 074034 (2003); M. Loewe and C. Villavicencio, arXiv:1107.3859; D. C. Duarte, R. L. S. Farias, and R. O. Ramos, Phys. Rev. D 84, 083525 (2011); D. Ebert, K. G. Klimenko, A. V. Tyukov, and V. C. Zhukovsky, Eur. Phys. J. C 58, 57 (2008).

[11] L. He, M. Jin, and P. Zhuang, Phys. Rev. D 71, 116001 (2005).

[12] D. Ebert and K. G. Klimenko, J. Phys. G 32, 599 (2006); Eur. Phys. J. C 46, 771 (2006).

[13] J. O. Andersen and T. Brauner, Phys. Rev. D 78, 014030 (2008); J. O. Andersen and L. Kyllingstad, J. Phys. G 37, 015003 (2010); Y. Jiang, K. Ren, T. Xia, and P. Zhuang, Eur. Phys. J. C 71, 1822 (2011); A. Folkestad and J. O. Andersen, Phys. Rev. D 99, 054006 (2019); P. Adhikari, J. O. Andersen, and P. Kneschke, arXiv:1904.03887; A. A. Garibli, R. G. Jafarov, and V. E. Rochev, Symmetry 11, 668 (2019); J. O. Andersen, P. Adhikari, and P. Kneschke, arXiv:1810.00419; P. Adhikari, J. O. Andersen, and P. Kneschke, Phys. Rev. D 98, 074016 (2018).

[14] C.f. Mu, L. y. He, and Y. x. Liu, Phys. Rev. D 82, 056006 (2010).

[15] H. Abuki, R. Anglani, R. Gatto, G. Nardulli, and M. Ruggieri, Phys. Rev. D 78, 034034 (2008); H. Abuki, R. Anglani, R. Gatto, M. Pellicoro, and M. Ruggieri, Phys.
Rev. D 79, 034032 (2009); R. Anglani, Acta Phys. Polon. Supp. 3, 735 (2010).

[16] D. Ebert, T. G. Khunjua, K. G. Klimenko, and V. C. Zhukovsky, Int. J. Mod. Phys. A 27, 1250162 (2012).

[17] N. V. Gubina, K. G. Klimenko, S. G. Kurbanov, and V. C. Zhukovsky, Phys. Rev. D 86, 085011 (2012).

[18] M. Thies, Phys. Rev. D 98, 096019 (2018).

[19] T. G. Khunjua, K. G. Klimenko, and R. N. ZhokhovLarionov, EPJ Web Conf. 191, 05016 (2018); T. G. Khunjua, V. C. Zhukovsky, K. G. Klimenko, and R. N. Zhokhov, Int. J. Mod. Phys. Conf. Ser. 47, 1860093 (2018); T. Khunjua, K. Klimenko, and R. Zhokhov, EPJ Web Conf. 191, 05015 (2018); T. G. Khunjua, K. G. Klimenko, R. N. Zhokhov, and V. C. Zhukovsky, Phys. Rev. D 95, 105010 (2017).

[20] A. Mammarella and M. Mannarelli, Phys. Rev. D 92, 085025 (2015).

[21] S. Carignano, L. Lepori, A. Mammarella, M. Mannarelli, and G. Pagliaroli, Eur. Phys. J. A 53, 35 (2017).

[22] T. G. Khunjua, K. G. Klimenko, and R. N. Zhokhov, Symmetry 11, 778 (2019).

[23] J. O. Andersen and P. Kneschke, arXiv:1807.08951; B. B. Brandt, G. Endrodi, E. S. Fraga, M. Hippert, J. SchaffnerBielich, and S. Schmalzbauer, Phys. Rev. D 98, 094510 (2018).

[24] K. Fukushima, D. E. Kharzeev, and H. J. Warringa, Phys. Rev. D 78, 074033 (2008).

[25] M. A. Metlitski and A. R. Zhitnitsky, Phys. Rev. D 72, 045011 (2005).

[26] T. G. Khunjua, K. G. Klimenko, and R. N. Zhokhov, Eur. Phys. J. C 79, 151 (2019).

[27] T. G. Khunjua, K. G. Klimenko, and R. N. Zhokhov, J. High Energy Phys. 06 (2019) 006.

[28] M. Ruggieri, G. X. Peng, and M. Chernodub, EPJ Web Conf. 129, 00037 (2016); M. Ruggieri and G. X. Peng, Phys. Rev. D 93, 094021 (2016).

[29] A. A. Andrianov, D. Espriu, and X. Planells, Eur. Phys. J. C 73, 2294 (2013); 74, 2776 (2014); R. Gatto and M. Ruggieri, Phys. Rev. D 85, 054013 (2012); L. Yu, H. Liu, and M. Huang, Phys. Rev. D 90, 074009 (2014); L. Yu, H. Liu, and M. Huang, Phys. Rev. D 94, 014026 (2016); G. Cao and P. Zhuang, Phys. Rev. D 92, 105030 (2015); M. Ruggieri and G. X. Peng, J. Phys. G 43, 125101 (2016).

[30] V. V. Braguta and A. Y. Kotov, Phys. Rev. D 93, 105025 (2016); V. V. Braguta, E. M. Ilgenfritz, A. Y. Kotov, B. Petersson, and S. A. Skinderev, Phys. Rev. D 93, 034509 (2016); N. Y. Astrakhantsev, V. V. Braguta, A. Y. Kotov, and A. A. Nikolaev, arXiv:1902.09325; V. V. Braguta, V. A. Goy, E. M. Ilgenfritz, A. Y. Kotov, A. V. Molochkov, M. Mller-Preussker, B. Petersson, and A. Schreiber, AIP Conf. Proc. 1701, 060002 (2016); V. V. Braguta, M. I. Katsnelson, A. Y. Kotov, and A. M. Trunin, arXiv:1904.07003.

[31] T. G. Khunjua, K. G. Klimenko, and R. N. Zhokhov, Phys. Rev. D 97, 054036 (2018); 98, 054030 (2018).

[32] D. J. Gross and A. Neveu, Phys. Rev. D 10, 3235 (1974).

[33] J. Feinberg, Ann. Phys. (Amsterdam) 309, 166 (2004); M. Thies, J. Phys. A 39, 12707 (2006).

[34] U. Wolff, Phys. Lett. B 157B, 303 (1985); T. Inagaki, T. Kouno, and T. Muta, Int. J. Mod. Phys. A 10, 2241 (1995); S. Kanemura and H.-T. Sato, Mod. Phys. Lett. A 10, 1777 (1995). 
[35] K. G. Klimenko, Theor. Math. Phys. 75, 487 (1988).

[36] A. Barducci, R. Casalbuoni, R. Gatto, M. Modugno, and G. Pettini, Phys. Rev. D 51, 3042 (1995).

[37] A. Chodos, H. Minakata, F. Cooper, A. Singh, and W. Mao, Phys. Rev. D 61, 045011 (2000); K. Ohwa, Phys. Rev. D 65, 085040 (2002).

[38] A. Chodos and H. Minakata, Phys. Lett. A 191, 39 (1994); H. Caldas, J. L. Kneur, M. B. Pinto, and R. O. Ramos, Phys. Rev. B 77, 205109 (2008); H. Caldas, J. Stat. Mech. (2011) P10005.

[39] V. Schon and M. Thies, Phys. Rev. D 62, 096002 (2000); A. Brzoska and M. Thies, Phys. Rev. D 65, 125001 (2002).

[40] N. D. Mermin and H. Wagner, Phys. Rev. Lett. 17, 1133 (1966); S. Coleman, Commun. Math. Phys. 31, 259 (1973).

[41] D. Ebert, K. G. Klimenko, A. V. Tyukov, and V. C. Zhukovsky, Phys. Rev. D 78, 045008 (2008).

[42] D. Ebert and K. G. Klimenko, Phys. Rev. D 80, 125013 (2009); V. C. Zhukovsky, K. G. Klimenko, and T. G. Khunjua, Moscow Univ. Phys. Bull. 65, 21 (2010).

[43] D. Ebert and K. G. Klimenko, arXiv:0902.1861.

[44] P. Adhikari and J. O. Andersen, Phys. Rev. D 95, 054020 (2017).

[45] D. Ebert, N. V. Gubina, K. G. Klimenko, S. G. Kurbanov, and V. C. Zhukovsky, Phys. Rev. D 84, 025004 (2011).

[46] V. P. Gusynin, V. A. Miransky, and I. A. Shovkovy, Phys. Lett. B 349, 477 (1995).
[47] M. Thies, Phys. Rev. D 68, 047703 (2003); 90, 105017 (2014).

[48] D. Ebert, T. G. Khunjua, K. G. Klimenko, and V.C. Zhukovsky, Phys. Rev. D 90, 045021 (2014); 93, 105022 (2016).

[49] M. Hanada and N. Yamamoto, J. High Energy Phys. 02 (2012) 138; Proc. Sci., LATTICE2011 (2011) 221.

[50] D. Ebert, T. G. Khunjua, and K. G. Klimenko, Phys. Rev. D 94, 116016 (2016).

[51] L. Jacobs, Phys. Rev. D 10, 3956 (1974); K. G. Klimenko, Teor. Mat. Fiz. 70, 125 (1987) [Theor. Math. Phys. 70, 87 (1987)].

[52] J. A. Pons, S. Reddy, M. Prakash, J. M. Lattimer, and J. A. Miralles, Astrophys. J. 513, 780 (1999); J. A. Pons, A. W. Steiner, M. Prakash, and J. M. Lattimer, Phys. Rev. Lett. 86, 5223 (2001); J. Roark and V. Dexheimer, Phys. Rev. C 98, 055805 (2018).

[53] T. Fischer et al., Phys. At. Nucl. 75, 613 (2012).

[54] A. Bauswein, H. T. Janka, K. Hebeler, and A. Schwenk, Phys. Rev. D 86, 063001 (2012).

[55] H. Zheng, G. Bonasera, J. Mabiala, P. Marini, and A. Bonasera, Eur. Phys. J. A 50, 167 (2014); V. Dexheimer, M. Hempel, I. Iosilevskiy, and S. Schramm, Nucl. Phys. A967, 780 (2017).

[56] G. Birkhoff and S. Mac Lane, A Survey of Modern Algebra (Macmillan, New York, 1977). 